\title{
SÍNTESE E AVALIAÇÃO DA ATIVIDADE CITOTÓXICA DE DERIVADOS DO EUGENOL CONTENDO NÚCLEOS 1,2,3-TRIAZÓLICOS
}

Poliana Aparecida Rodrigues Gazollaa, Róbson Ricardo Teixeira ${ }^{\mathrm{a}, *}$, Adalberto Manoel da Silva ${ }^{\mathrm{b}}$, Boniek Gontijo Vaz, Géssica Adriana Vasconcelos ${ }^{\mathrm{c}}$, Raoni Pais Siqueira ${ }^{\mathrm{d}}$, Victor Hugo Sousa Gonçalves ${ }^{\mathrm{d}}$, Higor Sette Pereira ${ }^{\mathrm{d}}$ e Gustavo Costa Bressand $^{\text {d }}$

aDepartamento de Química, Universidade Federal de Viçosa, 36570-900 Viçosa - MG, Brasil

'Instituto Federal de Educação, Ciência e Tecnologia Catarinense, 89245-000 Araquari - SC, Brasil

'Instituto de Química, Universidade Federal de Goiás, Av. Esperança, S/N, Campus Samambaia, 74690-970, Goiânia - GO, Brasil

¿Departamento de Bioquímica e Biologia Molecular, Universidade Federal de Viçosa, 36570-900 Viçosa - MG, Brasil

Recebido em 21/09/2017; aceito em 29/01/2018; publicado na web em 22/02/2018

\begin{abstract}
SYNTHESIS AND CYTOTOXIC ACTIVITY OF 1,2,3-TRIAZOLE DERIVATIVES OF EUGENOL. Eugenol is an aromatic compound found in several plant species. It presents important biological activities including cytotoxicity. In this paper, it is described the synthesis and the evaluation of the cytotoxic activity of eugenol derivatives bearing 1,2,3-triazole functionalities. Eugenol, extracted via hydrodistillation from dried flower buds of Eugenia caryophyllata (=Syzygium aromaticum), was submitted to alkylation reactions to afford two terminal alkynes in good yields. The key reaction involved in the preparation of eugenol derivatives corresponded to the Copper(I)-catalyzed Azide-Alkyne Cycloaddition (CuAAC), between alkynylated eugenol derivatives and different benzyl azides. The evaluation of the cytotoxicity of twenty seven synthesized derivatives against HL60 leukemia cell line revealed that at $100 \mu \mathrm{mol} \mathrm{L}{ }^{-1}$, five of them, namely 4-((4-allyl-2-methoxyphenoxy)methyl)-1-(3-bromobenzyl)-1H-1,2,3triazole (6n), 4-(3-(4-allyl-2-methoxyphenoxy)propyl)-1-benzyl-1 H-1,2,3-triazole (7a), 4-(3-(4-allyl-2-methoxyphenoxy)propyl)1-(4-chlorobenzyl)-1H-1,2,3-triazole (7c), 4-(3-(4-allyl-2-methoxyphenoxy)propyl)-1-(4-iodobenzyl)-1H-1,2,3-triazole (7e) and 4-(3-(4-allyl-2-methoxyphenoxy)propyl)-1-(3-bromobenzyl)-1H-1,2,3-triazole (7m), were capable of significantly decreasing cell viability. These most active triazolic derivatives were also evaluated against B16F10 melanoma and Nalm6 leukemia cell lines. While only compound $\mathbf{7 a}$ was active against the former, compounds $\mathbf{6 n}, \mathbf{7 a}$, and $\mathbf{7} \mathbf{m}$ displayed activity against the latter. Derivative $7 \mathbf{a}$ was active against all cell lines. It is believed that eugenol derivatives bearing triazole functionalities may represent a scaffold to be explored toward the development of new agents against cancer.
\end{abstract}

Keywords: eugenol; triazole; cytotoxic activity; click chemistry; CuAAC reaction.

\section{INTRODUÇÃO}

O câncer correspondeu a uma das principais causas de mortes no século XX e vem difundindo-se de forma contínua e aumentando sua incidência no século XXI. ${ }^{1}$ Sua denominação está associada a um conjunto de mais de 100 doenças que têm em comum o crescimento desordenado (maligno) de células anormais que invadem os tecidos e órgãos, podendo espalhar-se (metástase) para outras regiões do corpo. Devido à rápida divisão celular, estas células podem se tornar muito agressivas e incontroláveis, determinando assim a formação de tumores ou neoplasias malignas.

De acordo com a Organização Mundial da Saúde (OMS), o câncer é um problema de saúde pública, principalmente em países em desenvolvimento. A estimativa para o ano de 2025 aponta para a ocorrência de aproximadamente 20 milhões de novos casos. ${ }^{2}$

Investigações científicas multidisciplinares têm sido conduzidas buscando encontrar alternativas para combater esta doença, no entanto, a cura ainda não foi alcançada. Os principais tipos de tratamento empregados para o câncer baseiam-se, de forma geral, na associação da ressecção cirúrgica dos tumores ao tratamento radioterápico e a quimioterapia.

No que tange à quimioterapia, são utilizados compostos químicos que possuem como objetivo primário a destruição das células neoplásicas e a preservação das células saudáveis. ${ }^{3}$

Dentre os diversos medicamentos quimioterápicos para o

*e-mail: robsonr.teixeira@ufv.br tratamento do câncer introduzidos nas últimas décadas, a maioria $(60 \%)$ tem sua origem nos produtos naturais. Como exemplo, mencionam-se os quimioterápicos vimblastina $\left(\right.$ Velban $\left.^{\circledR}\right)$, vincristina $\left(\right.$ Oncovin $\left.{ }^{\circledR}\right)$, paclitaxel $\left(\operatorname{Taxol}^{\circledR}\right)$, podofilotoxina, etoposídeo (Etopophos ${ }^{\circledR}$ ) e camptotecina (Figura 1). ${ }^{4}$

Apesar da introdução de diversos fármacos no arsenal terapêutico contra o câncer, a quimioterapia apresenta diversos problemas dentre os quais se destacam os efeitos colaterais (como diarréia, vômito, fraqueza, queda de cabelo e tonturas) e a resistência intrínseca ou adquirida de diversos tipos de câncer aos agentes quimioterápicos. ${ }^{5,6}$ Devido a estes fatores, a exploração de produtos naturais pela busca do desenvolvimento de novos quimioterápicos, mais específicos e eficientes, continua sendo prioridade de investigação de diversos pesquisadores. ${ }^{7-10}$

O eugenol (1) (Figura 2) é um composto natural encontrado em várias plantas aromáticas como noz moscada, canela, folha de louro, sassafrás, mirra e manjericão. ${ }^{11}$ No entanto, o cravo da índia Eugenia caryophyllata (=Syzygium aromaticum) pode ser considerado a principal fonte natural do eugenol (1), pois representa entre $45 \%$ e $90 \%$ da composição do óleo essencial derivado desta espécie.

Várias atividades biológicas foram relatadas para o eugenol (1), dentre elas destacam-se as atividades antibacteriana, antifúngica, antiplasmodial, antiviral, anti-inflamatória, analgésica, antioxidante, antidiabetes e citotóxica contra diferentes linhagens celulares de câncer. ${ }^{12,13}$ Uma investigação realizada sobre o efeito da atividade antiproliferativa do eugenol (1) contra as linhagens de células leucêmicas U-937, HL-60, HepG2, 3LL Lewis e SNU-C5, resultaram em valores de $\mathrm{IC}_{50}$ iguais a 39,4, 23,7, 118,6, 89,6 e 129,4 $\mu \mathrm{mol} \mathrm{L} \mathrm{L}^{-1}$, 


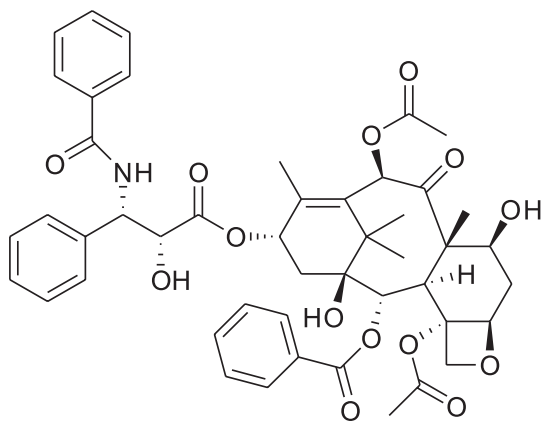

Paclitaxel<smiles>COc1cc([C@H]2c3cc4c(cc3[C@@H](O)[C@@H]3COC(=O)[C@H]23)OCO4)cc(OC)c1OC</smiles>

Podofilotoxina<smiles>COc1cc([C@H]2c3cc4c(cc3[C@@H](OC3OC5COC(C)OC(CO)(C5)O3)[C@H]3COC(=O)[C@H]23)OCO4)cc(OC)c1OC</smiles>

Etoposídeo

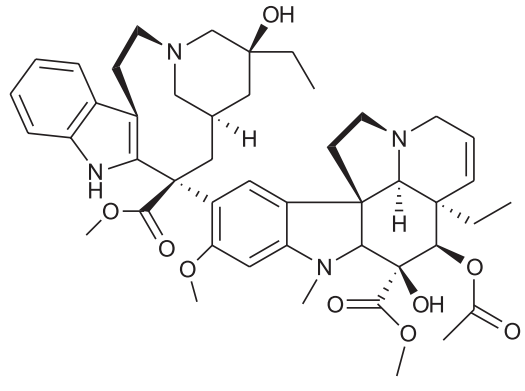

Vimblastina

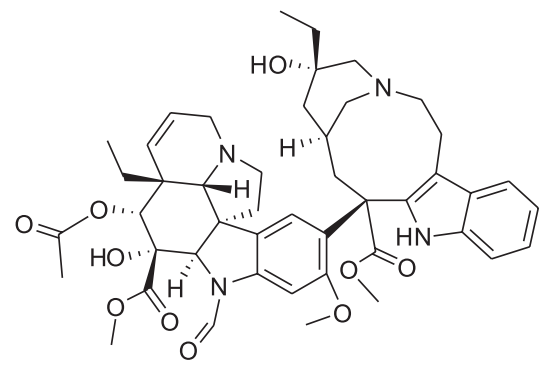

Vincristina<smiles>CC[C@]1(O)C(=O)OCc2c1cc1n(c2=O)Cc2cc3ccccc3nc2-1</smiles>

Camptotecina

Figura 1. Estruturas de alguns quimioterápicos originários de plantas e empregados no tratamento do câncer<smiles>C=CCc1ccc(O)c(OC)c1</smiles>

Figura 2. Estrutura do eugenol (1)

respectivamente. ${ }^{14}$

Considerando o efeito citotóxico descrito para o eugenol (1) bem como diversos trabalhos descritos na literatura a respeito da atividade citotóxica de compostos 1,2,3- triazólicos, é descrito neste artigo a síntese e a avaliação da atividade citotóxica de uma série de compostos derivados do eugenol (1) contendo núcleos 1,2,3-triazólicos.

\section{PARTE EXPERIMENTAL}

\section{Generalidades}

Os reagentes pent-4-in-1-ol, cloreto de mesila, azida de sódio, trietilamina, brometo de propargila, sulfato de cobre pentaidratado e os álcoois benzílicos foram adquiridos comercialmente da Sigma Aldrich (St. Louis, MO, United States) e utilizados sem prévia purificação. Os solventes foram adquiridos comercialmente da Vetec (Rio de Janeiro, Brasil). A secagem dos solventes foi realizada utilizando procedimentos padrão descritos na literatura. ${ }^{15} \mathrm{O}$ eugenol (1) foi extraído por meio da hidrodestilação de cravos da índia adquiridos no mercado local (Viçosa, Minas Gerais, Brasil) e purificado por cromatografia em coluna de sílica gel 60 (70-230 mesh-ASTM, Merck). Os espectros de $\mathrm{RMN}{ }^{1} \mathrm{H}(300 \mathrm{MHz}) \mathrm{e}^{13} \mathrm{C}(75 \mathrm{MHz})$ foram obtidos em um instrumento Varian Mercury 300 utilizando $\mathrm{CDCl}_{3} \mathrm{e}$ $\mathrm{C}_{6} \mathrm{D}_{6}$ como solventes. Os dados de RMN são apresentados da seguinte forma: deslocamento químico $(\delta)$ em ppm, multiplicidade, número de hidrogênios, valores de $J$ em Hertz (Hz). As multiplicidades são apresentadas como as seguintes abreviaturas: s (simpleto), sl (simpleto largo), d (dupleto), dd (duplo dupleto), ddt ap $_{\text {(duplo duplo }}$ tripleto aparente), t (tripleto), dt (duplo tripleto), q (quarteto), quint (quinteto), m (multipleto). Os espectros no IV foram obtidos em equipamento Varian 660-IV equipado com GladiATR na região de 4000 a $500 \mathrm{~cm}^{-1}$. As análises por cromatografia em camada delgada (CCD) foram realizadas em placas de sílica gel pré-revestidas com suporte de alumínio, usando diferentes sistemas de solventes e foram visualizadas usando solução de permanganato de potássio e/ou luz UV. Os pontos de fusão foram determinados em aparelho MQAPF-302 e não foram corrigidos. Os espectros de massa de baixa resolução foram obtidos em equipamento CG-EM SHIMADZU GCMS-QP5050A sob condições de impacto de elétrons $(70 \mathrm{eV})$. Os espectros de massas de alta resolução foram obtidos em equipamento Q-Exactive (Thermo Scientific, Bremen, Alemanha). Foram empregadas as seguintes condicões: Fonte de ionização: elestrospray (+) e (-); spray voltage: $3.5 \mathrm{kV}$; temperatura do capilar: $275^{\circ} \mathrm{C}$; sheath gas: 5 (unidades arbitrárias); auxilary gas: 0 (unidades arbitrárias). Para obtenção dos espectros de massas de alta resolução as amostras para análise foram preparadas da seguinte maneira: uma massa de $1 \mathrm{mg}$ das amostras foi solubilizada em $1 \mathrm{~mL}$ de acetonitrila e diluídas em metanol até a concentração final de $1 \mathrm{ppm}$. A solução resultante foi diretamente injetada no equipamento Q-Exactive a um fluxo de $5 \mu \mathrm{L} \mathrm{min}{ }^{-1}$. Os espectros foram adquiridos no modo full-MS.

\section{Extração e purificação do eugenol (1)}

O Eugenol (1) foi extraído via hidrodestilação de brotos secos de Eugenia caryophyllata (=Syzygium aromaticum), conhecidos como cravo-da-índia. Assim, 60,0 g de cravo e $500 \mathrm{~mL}$ de água destilada foram misturados em um balão de fundo redondo que foi conectado ao sistema de hidrodestilação. A mistura foi aquecida 
durante três horas. O hidrolato obtido foi transferido para um funil de separação e a fase aquosa extraída com diclorometano $(3$ x $30 \mathrm{~mL})$. Os extratos orgânicos foram reunidos e a fase orgânica resultante foi seca com sulfato de sódio anidro, filtrada e concentrada sob pressão reduzida. O óleo resultante foi submetido à purificação por cromatografia em coluna de sílica gel eluída com hexano-acetato de etila $(6: 1 \mathrm{v} / \mathrm{v})$. O procedimento descrito proporcionou a obtenção de $7,12 \mathrm{~g}$ de eugenol (1) que correspondeu a aproximadamente $12 \% \mathrm{de}$ rendimento em relação à massa inicial de cravo da índia utilizado no processo de extração. A estrutura do eugenol (1) está de acordo com os seguintes dados.

Óleo amarelo, $\mathrm{R}_{\mathrm{f}}=0,44$ (hexano-acetato de etila $6: 1 \mathrm{v} / \mathrm{v}$ ). $\mathrm{RMN}{ }^{1} \mathrm{H}$ $\left(300 \mathrm{MHz}, \mathrm{CDCl}_{3}\right) \delta: 3,33(\mathrm{~d}, 2 \mathrm{H}, J=6,7 \mathrm{~Hz}) ; 3,88(\mathrm{~s}, 3 \mathrm{H}) ; 5,02-5,13$ $(\mathrm{m}, 2 \mathrm{H}) ; 5,54(\mathrm{~s}, 1 \mathrm{H}, \mathrm{OH}) ; 5,97\left(\mathrm{ddt}_{\mathrm{ap}}, 1 \mathrm{H}, J_{l}=16,8 \mathrm{~Hz}, J_{2}=10,2 \mathrm{~Hz}\right.$ e $\left.J_{3}=6,7 \mathrm{~Hz}\right) ; 6,67-6,72(\mathrm{~m}, 2 \mathrm{H}) ; 6,86(\mathrm{~d}, 1 \mathrm{H}, J=8,5 \mathrm{~Hz}) . \mathrm{RMN}{ }^{13} \mathrm{C}$ $\left(75 \mathrm{MHz}, \mathrm{CDCl}_{3}\right.$ ) $\delta: 39,8 ; 55,8 ; 111,1 ; 114,2 ; 115,5 ; 121,1 ; 131,9$; 137,$8 ; 143,8 ; 146 . \mathrm{EM}, \mathrm{m} / z(\%): 164\left([\mathrm{M}] \pm, \mathrm{C}_{10} \mathrm{H}_{12} \mathrm{O}_{2}, 100\right), 149(35)$, 131 (29), 103 (32), 91 (28), 77 (34), 65 (13), 55 (24), 41 (7).

\section{Procedimentos sintéticos}

Sintese do 4-alil-2-metoxi-1-(prop-2-in-1-iloxi)benzeno (2)

A um balão de fundo redondo de $50 \mathrm{~mL}$ foram adicionados hidróxido de sódio (0,313 g; 7,38 mmol), eugenol (1) (1,20 g; 7,32 mmol) e $25,0 \mathrm{~mL}$ de metanol. A mistura resultante foi aquecida a $40^{\circ} \mathrm{C}$ e mantida sob agitação magnética por 30 minutos. Em seguida, o metanol foi removido sob pressão reduzida e $10,0 \mathrm{~mL}$ de etanol anidro foram adicionados para a eliminação de água residual. O etanol foi removido e ao balão, sob atmosfera de nitrogênio, adicionaram-se acetonitrila ( $25 \mathrm{~mL})$ e brometo de propargila $(800 \mu \mathrm{L} ; 8,79 \mathrm{mmol})$ lentamente. O sistema foi mantido sob agitação à temperatura ambiente por 18 horas. Após esse período, foi evidenciado através da análise por CCD o término da reação. A mistura de reação foi concentrada e particionada com 25,0 mL de solução aquosa $\left(0,1 \mathrm{~mol} \mathrm{~L}^{-1}\right)$ de hidróxido de sódio e éter etílico $(3 \times 25,0 \mathrm{~mL})$. As fases orgânicas foram reunidas, lavadas com solução aquosa saturada de cloreto de sódio $(25,0 \mathrm{~mL})$, seca com sulfato de sódio anidro, filtrada e concentrada sob pressão reduzida. O produto resultante foi purificado por cromatografia em coluna de sílica gel eluída com hexano-acetato de etila (6:1 v/v) e obtido com $87 \%$ (1,29 g; 6,37 mmol) de rendimento. A estrutura do composto 2 está de acordo com os seguintes dados.

Óleo amarelo, $\mathrm{R}_{\mathrm{f}}=0,45$ (hexano-acetato de etila $6: 1 \mathrm{v} / \mathrm{v}$ ). $\mathrm{RMN}{ }^{1} \mathrm{H}\left(300 \mathrm{MHz}, \mathrm{CDCl}_{3}\right) \delta: 2,49(\mathrm{t}, 1 \mathrm{H}, J=2,4 \mathrm{~Hz}) ; 3,35(\mathrm{~d}$, $2 \mathrm{H}, J=6,7 \mathrm{~Hz}) ; 3,86(\mathrm{~s}, 3 \mathrm{H}) ; 4,73(\mathrm{~d}, 2 \mathrm{H}, J=2,4 \mathrm{~Hz}) ; 5,03-5,14$ $(\mathrm{m}, 2 \mathrm{H}) ; 5,96\left(\mathrm{ddt}_{\mathrm{ap}}, 1 \mathrm{H}, J_{1}=16,8 \mathrm{~Hz}, J_{2}=10,2 \mathrm{~Hz}, J_{3}=6,7 \mathrm{~Hz}\right)$; 6,70-6,76 (m, 2H); 6,97 (d, 1H, $J=8,6 \mathrm{~Hz})$. RMN ${ }^{1} \mathrm{H}(300 \mathrm{MHz}$, $\left.\mathrm{C}_{6} \mathrm{D}_{6}\right) \delta: 1,99(\mathrm{t}, 1 \mathrm{H}, J=2,4 \mathrm{~Hz}) ; 3,14(\mathrm{~d}, 2 \mathrm{H}, J=6,6 \mathrm{~Hz}) ; 3,31(\mathrm{~s}$, $3 \mathrm{H}) ; 4,36(\mathrm{~d}, 2 \mathrm{H}, J=2,4 \mathrm{~Hz}) ; 4,93-5,02(\mathrm{~m}, 2 \mathrm{H}) ; 5,85\left(\mathrm{ddt}_{\mathrm{ap}}, 1 \mathrm{H}\right.$, $J_{l}=17,0 \mathrm{~Hz}, J_{2}=10,4 \mathrm{~Hz}$ e $\left.J_{3}=6,6 \mathrm{~Hz}\right) ; 6,52(\mathrm{~d}, 1 \mathrm{H}, J=1,7 \mathrm{~Hz})$; $6,57\left(\mathrm{dd}, 1 \mathrm{H}, J_{I}=8,1 \mathrm{~Hz}\right.$ e $\left.J_{2}=1,7 \mathrm{~Hz}\right) ; 6,83(\mathrm{~d}, 1 \mathrm{H}, J=8,1 \mathrm{~Hz})$. $\mathrm{RMN}{ }^{13} \mathrm{C}\left(75 \mathrm{MHz}, \mathrm{CDCl}_{3}\right) \delta: 39,8 ; 55,8 ; 56,9 ; 75,5 ; 78,7 ; 112,3$; 114,$6 ; 115,7 ; 120,3 ; 134,2 ; 137,4 ; 145,0 ; 149,6$. EM, $m / z(\%): 202$ ([M]+, $\left.\mathrm{C}_{13} \mathrm{H}_{14} \mathrm{O}_{2}, 40\right), 163$ (100), 135 (9), 103 (42), 91 (33), 77 (19), 65 (13), 55 (9), 41 (23).

\section{Síntese do pent-4-in-1-ilmetanosulfonato (3)}

A um balão de fundo redondo de $100 \mathrm{~mL}$ foram adicionados pent-4-in-1-ol (1,68 g, 20,0 mmol) e diclorometano (20 mL). A mistura de reação, sob atmosfera de nitrogênio, foi resfriada a $-50{ }^{\circ} \mathrm{C}$ e trietilamina $(5,60 \mathrm{~mL}, 40,0 \mathrm{mmol})$ foi adicionada. Em seguida, adicionou-se lentamente ao meio reacional cloreto de mesila $(2,3 \mathrm{~mL}$, $30,0 \mathrm{mmol}$ ). Após o término da reação, evidenciado por análise de CCD, adicionaram-se $10,0 \mathrm{~mL}$ de água destilada. A fase orgânica foi extraída, lavada com solução aquosa de $\mathrm{HCl} 1 \%(3 \times 15,0 \mathrm{~mL})$ seguida de solução aquosa saturada de $\mathrm{NaHCO}_{3}(3 \times 5,0 \mathrm{~mL})$, seca com sulfato de sódio anidro, filtrada e concentrada sobre pressão reduzida. O produto foi purificado por cromatografia em coluna de sílica gel eluída com hexano-acetato de etila-diclorometano (3:1:3 $\mathrm{v} / \mathrm{v})$ e obtido com $92 \%$ (3,00 g, 18,0 mmol) de rendimento. A estrutura do composto 3 está de acordo com os seguintes dados.

Óleo amarelo, $\mathrm{R}_{\mathrm{f}}=0,76$ (hexano-acetato de etila-diclorometano 3:1:3 v/v). RMN ${ }^{1} \mathrm{H}\left(300 \mathrm{MHz}, \mathrm{CDCl}_{3}\right) \delta: 1,93$ (quint, $2 \mathrm{H}$, $J=6,5 \mathrm{~Hz}) ; 1,99(\mathrm{t}, 1 \mathrm{H}, J=2,7 \mathrm{~Hz}) ; 2,33\left(\mathrm{dt}, 2 \mathrm{H}, J_{l}=6,8 \mathrm{~Hz}\right.$ e $\left.J_{2}=2,7 \mathrm{~Hz}\right) ; 3,00\left(\mathrm{~s}, 3 \mathrm{H}, \mathrm{C}_{3}\right) ; 4,32(\mathrm{t}, 2 \mathrm{H}, J=6,1 \mathrm{~Hz}) . \mathrm{RMN}{ }^{13} \mathrm{C}$ $\left(75 \mathrm{MHz}, \mathrm{CDCl}_{3}\right) \delta: 14,5 ; 27,6 ; 37,1 ; 68,2 ; 69,7 ; 82,0$.

\section{Sintese do 4-alil-2-metoxi-1-(pent-4-in-1-iloxi)benzeno (4)}

Este composto foi preparado utilizando um procedimento semelhante ao descrito para a preparação do composto 2. Neste caso, o agente de alquilação correspondeu ao composto 3 . A substância 4 foi obtida com $78 \%$ de rendimento (1,64 g, 7,13 mmol) após purificação por cromatografia em coluna de sílica gel eluída com hexano-acetato de etila $(6: 1 \mathrm{v} / \mathrm{v})$. A estrutura de 4 é suportada pelos seguintes dados.

Óleo amarelo, $\mathrm{R}_{\mathrm{f}}=0,55$ (hexano-acetato de etila $6: 1 \mathrm{v} / \mathrm{v}$ ). $\mathrm{RMN}{ }^{1} \mathrm{H}\left(300 \mathrm{MHz}, \mathrm{CDCl}_{3}\right) \delta: 1,97(\mathrm{t}, 1 \mathrm{H}, J=2,6 \mathrm{~Hz}) ; 2,04$ (quint, $2 \mathrm{H}, J=6,6 \mathrm{~Hz}) ; 2,42\left(\mathrm{dt}, 2 \mathrm{H}, J_{1}=7,0 \mathrm{~Hz}\right.$ e $\left.J_{2}=2,6 \mathrm{~Hz}\right) ; 3,34(\mathrm{~d}, 2 \mathrm{H}$, $J=6,6 \mathrm{~Hz}) ; 3,85(\mathrm{~s}, 3 \mathrm{H}) ; 4,10(\mathrm{t}, 2 \mathrm{H}, J=6,3 \mathrm{~Hz}) ; 5,03-5,13(\mathrm{~m}, 2 \mathrm{H})$; $5,97\left(\mathrm{ddt}_{\mathrm{ap}}, 1 \mathrm{H}, J_{l}=16,8 \mathrm{~Hz}, J_{2}=10,2 \mathrm{~Hz}\right.$ e $\left.J_{3}=6,7 \mathrm{~Hz}\right) ; 6,68-6,75$ $(\mathrm{m}, 2 \mathrm{H}) ; 6,85(\mathrm{~d}, 1 \mathrm{H}, J=8,7 \mathrm{~Hz}) . \mathrm{RMN}{ }^{13} \mathrm{C}\left(75 \mathrm{MHz}, \mathrm{CDCl}_{3}\right) \delta$ : 15,$2 ; 28,2 ; 39,8 ; 55,9 ; 67,5 ; 68,8 ; 83,6 ; 112,4 ; 113,6 ; 115,6 ; 120,5$; 133,0; 137,6; 146,7; 149,5. EM, m/z (\%): 230 ([M]", $\left.\mathrm{C}_{15} \mathrm{H}_{18} \mathrm{O}_{2}, 100\right)$, 164 (68), 149 (44), 131 (29), 121 (16), 103 (34), 91 (31), 77 (23), 65 (16), 55 (15), 41 (34).

Sintese dos compostos 6a-6n e 7a-7m exemplificados pela síntese do composto 4 - ((4-alil-2-metoxifenoxi)metil)-1-benzil-1H-1,2,3triazole $(6 a)$

A um balão de fundo redondo de $10 \mathrm{~mL}$ foram adicionados 4-alil-2-metoxi-1-(prop-2-in-1-iloxi)benzeno (2) (0,150 g; 0,740 mmol), benzilazida $(0,0990 \mathrm{~g} ; 0,740 \mathrm{mmol})$, ascorbato de sódio $(0,0590 \mathrm{~g}$; $0,300 \mathrm{mmol}), 1,00 \mathrm{~mL}$ de etanol e 1,00 $\mathrm{mL}$ de água. Em seguida, adicionou-se $\mathrm{CuSO}_{4} \cdot 5 \mathrm{H}_{2} \mathrm{O}(0,0370 \mathrm{~g} ; 0,150 \mathrm{mmol})$. A mistura de reação foi agitada vigorosamente à temperatura ambiente. Após o término da reação evidenciado pela análise por $\mathrm{CCD}$, a mistura de reação foi extraída com diclorometano ( 3 x 10,0 mL). As fases orgânicas foram reunidas, lavadas com solução saturada de carbonato de sódio, seca com sulfato de sódio anidro, filtrada e concentrada sobre pressão reduzida. $\mathrm{O}$ composto $\mathbf{6} \mathbf{a}$ foi purificado por cromatografia em coluna de sílica gel eluída com hexano-acetato de etila-diclorometano (3:1:3 $\mathrm{v} / \mathrm{v})$. O procedimento descrito resultou na obtençao de $\mathbf{6 a}$ com $91 \%$ de rendimento $(0,228 \mathrm{~g} ; 0,680 \mathrm{mmol})$. A estrutura do composto $\mathbf{6 a}$ está de acordo com os seguintes dados.

Sólido branco, P.f. $=90,5-91,3^{\circ} \mathrm{C}, \mathrm{R}_{\mathrm{f}}=0,49$ (hexano-acetato de etila-diclorometano 3:1:3 v/v). IV (ATR) $v_{\max } / \mathrm{cm}^{-1}: 3086,3034,2999$, 2929, 2912, 1589, 1511, 1446, 1259, 1229, 1143, 1016, 921, 831, 713, 696. RMN ${ }^{1} \mathrm{H}\left(300 \mathrm{MHz}, \mathrm{CDCl}_{3}\right) \delta: 3,32(\mathrm{~d}, 2 \mathrm{H}, J=6,7 \mathrm{~Hz})$; $3,81(\mathrm{~s}, 3 \mathrm{H}) ; 5,02-5,12(\mathrm{~m}, 2 \mathrm{H}) ; 5,23(\mathrm{~s}, 2 \mathrm{H}) ; 5,50(\mathrm{~s}, 2 \mathrm{H}) ; 5,94\left(\mathrm{ddt}_{\mathrm{ap}}\right.$, $1 \mathrm{H}, J_{1}=16,9 \mathrm{~Hz}, J_{2}=10,2 \mathrm{~Hz}$ e $\left.J_{3}=6,7 \mathrm{~Hz}\right) ; 6,65-6,71(\mathrm{~m}, 2 \mathrm{H})$; $6,94(\mathrm{~d}, 1 \mathrm{H}, J=8,0 \mathrm{~Hz}) ; 7,21-7,40(\mathrm{~m}, 5 \mathrm{H}) ; 7,54(\mathrm{~s}, 1 \mathrm{H}) . \mathrm{RMN}{ }^{13} \mathrm{C}$ $\left(75 \mathrm{MHz}, \mathrm{CDCl}_{3}\right) \delta: 39,8 ; 54,1 ; 55,7 ; 63,5 ; 112,2 ; 114,6 ; 115,7$; 120,$4 ; 122,7 ; 128,1 ; 128,7 ; 129,0 ; 133,8 ; 134,4 ; 137,4 ; 144,8 ; 145,8$; 149,5. HRMS $\left(\mathrm{M}+\mathrm{H}^{+}\right)$: calculado para $\mathrm{C}_{20} \mathrm{H}_{22} \mathrm{~N}_{3} \mathrm{O}_{2}: 336,17120$; encontrado: 336.17020. HRMS $\left(\mathrm{M}+\mathrm{Na}^{+}\right)$: calculado para $\mathrm{C}_{20} \mathrm{H}_{21} \mathrm{~N}_{3} \mathrm{NaO}_{2}$ : 358,15315; encontrado: 358,15164 .

Os compostos $\mathbf{6 b}-\mathbf{6 n}$ e $\mathbf{7 a - 7} \mathbf{m}$ foram preparados utilizando um procedimento semelhante ao descrito para a síntese de $\mathbf{6 a}$. As 
estruturas destes compostos foram são suportadas pelos seguintes dados.

Dados para 4-((4-alil-2-metoxifenoxi)metil)-1-(4-fluorobenzil)-1H-1,2,3-triazol (6b): Sólido branco, purificado por cromatografia em coluna eluída com hexano-acetato de etila-diclorometano $(3: 1: 3 \mathrm{v} / \mathrm{v})$, P.f. $=92,1-92,7^{\circ} \mathrm{C}, \mathrm{R}_{\mathrm{f}}=0,39$ (hexano-acetato de etila-diclorometano $3: 1: 3 \mathrm{v} / \mathrm{v})$. IV (ATR) $v_{\max } / \mathrm{cm}^{-1}: 3069,3005,2935,2914,1588,1511$, 1259, 1223, 1143, 1017, 905, 771. RMN ${ }^{1} \mathrm{H}\left(300 \mathrm{MHz}, \mathrm{CDCl}_{3}\right) \delta$ : $3,32$ (d, 2H, $J=6,7 \mathrm{~Hz}) ; 3,81$ (s, 3H); 5,02-5, 12 (m, 2H); 5,23 (s, 2H); $5,47(\mathrm{~s}, 2 \mathrm{H}) ; 5,94\left(\mathrm{ddt}_{\mathrm{ap}}, 1 \mathrm{H}, J_{l}=17,0 \mathrm{~Hz}, J_{2}=10,5 \mathrm{~Hz} J_{3}=6,7 \mathrm{~Hz}\right)$; 6,67-6,70 (m, 2H); 6,93 (d, 1H, $J=7,9 \mathrm{~Hz}) ; 7,04\left(\mathrm{t}_{\mathrm{ap}}, 2 \mathrm{H}, J=8,6 \mathrm{~Hz}\right)$; 7,20-7,30 (m, 2H); 7,54 (s, 1H). $\mathrm{RMN}^{13} \mathrm{C}\left(75 \mathrm{MHz}, \mathrm{CDCl}_{3}\right)$ 8: 39,7; $53,4 ; 55,7 ; 63,4 ; 112,2 ; 114,5 ; 115,7 ; 116,1$ (d, $J=21,8 \mathrm{~Hz}) ; 120,4$; $122,6 ; 129,9$ (d, $J=8,3 \mathrm{~Hz}) ; 130,3$ (d, $J=3,0 \mathrm{~Hz}) ; 133,8 ; 137,4 ; 145,0$; 145,$8 ; 149,5 ; 162,8(\mathrm{~d}, J=246,0 \mathrm{~Hz})$. HRMS $\left(\mathrm{M}+\mathrm{H}^{+}\right)$: calculado para $\mathrm{C}_{20} \mathrm{H}_{21} \mathrm{FN}_{3} \mathrm{O}_{2}$ : 354,16178; encontrado: 354,16077. HRMS $\left(\mathrm{M}+\mathrm{Na}^{+}\right)$: calculado para $\mathrm{C}_{20} \mathrm{H}_{20} \mathrm{FN}_{3} \mathrm{NaO}_{2}$ : 376,14372; encontrado: 376,14240 .

Dados para 4-((4-alil-2-metoxifenoxi)metil)-1-(4-clorobenzil)-1H-1,2,3-triazol (6c): Sólido branco, purificado por cromatografia em coluna eluída com hexano-acetato de etila-diclorometano (3:1:3 v/v), P.f. $=112,3-112,6{ }^{\circ} \mathrm{C}, \mathrm{R}_{\mathrm{f}}=0,37$ (hexano-acetato de etila-diclorometano 3:1:3 v/v). IV (ATR) $v_{\max } / \mathrm{cm}^{-1}: 3065,3001,2936$, 2915, 1588, 1513, 1943, 1258, 1228, 1143, 1016, 907, 803, 722, 693, 592. $\mathrm{RMN}^{1} \mathrm{H}\left(300 \mathrm{MHz}, \mathrm{CDCl}_{3}\right) \delta: 3,32(\mathrm{~d}, 2 \mathrm{H}, J=6,7 \mathrm{~Hz}) ; 3,81$ (s, $3 \mathrm{H}) ; 5,02-5,12(\mathrm{~m}, 2 \mathrm{H}) ; 5,23(\mathrm{~s}, 2 \mathrm{H}) ; 5,47(\mathrm{~s}, 2 \mathrm{H}) ; 5,94\left(\mathrm{ddt}_{\mathrm{ap}}, 1 \mathrm{H}\right.$, $J_{1}=17,0 \mathrm{~Hz}, J_{2}=10,2 \mathrm{~Hz}$ e $\left.J_{3}=6,7 \mathrm{~Hz}\right) ; 6,65-6,73(\mathrm{~m}, 2 \mathrm{H}) ; 6,93(\mathrm{~d}$, $1 \mathrm{H}, J=7,9 \mathrm{~Hz}) ; 7,18(\mathrm{~d}, 2 \mathrm{H}, J=8,5 \mathrm{~Hz}) ; 7,33(\mathrm{~d}, 2 \mathrm{H}, J=8,5 \mathrm{~Hz}) ; 7,54$ (s, $1 \mathrm{H}) . \mathrm{RMN}^{13} \mathrm{C}\left(75 \mathrm{MHz}, \mathrm{CDCl}_{3}\right) \delta: 39,8 ; 53,4 ; 55,7 ; 63,4 ; 112,2$; 114,$6 ; 115,7 ; 120,4 ; 122,7 ; 129,2 ; 129,3 ; 132,9 ; 133,9 ; 134,7 ; 137,4$; 145,$0 ; 145,8 ; 149,5$. HRMS $\left(\mathrm{M}+\mathrm{H}^{+}\right)$: calculado para $\mathrm{C}_{20} \mathrm{H}_{21} \mathrm{ClN}_{3} \mathrm{O}_{2}$ : 370,13223; encontrado: 370,13132. HRMS $\left(\mathrm{M}+\mathrm{Na}^{+}\right)$: calculado para $\mathrm{C}_{20} \mathrm{H}_{20} \mathrm{ClN}_{3} \mathrm{NaO}_{2}$ : 392,11417; encontrado: 392,11285.

Dados para 4-((4-alil-2-metoxifenoxi)metil)-1-(4-bromobenzil)-1H-1,2,3-triazol (6d): Sólido branco, purificado por cromatografia em coluna eluída com hexano-acetato de etila-diclorometano (3:1:3 v/v), P.f. $=119,1-120,2{ }^{\circ} \mathrm{C}, \mathrm{R}_{\mathrm{f}}=0,59$ (hexano-acetato de etila-diclorometano 3:1:3 v/v). IV (ATR) $v_{\max } / \mathrm{cm}^{-1}: 3065,3001,2935$, 2915, 1588, 1513, 1385, 1258, 1229, 1144, 1015, 803, 738, 592, 488. RMN ${ }^{1} \mathrm{H}\left(300 \mathrm{MHz}, \mathrm{CDCl}_{3}\right) \delta: 3,32(\mathrm{~d}, 2 \mathrm{H}, J=6,7 \mathrm{~Hz}) ; 3,82$ $(\mathrm{s}, 3 \mathrm{H}) ; 5,02-5,12(\mathrm{~m}, 2 \mathrm{H}) ; 5,23(\mathrm{~s}, 2 \mathrm{H}) ; 5,45(\mathrm{~s}, 2 \mathrm{H}) ; 5,94\left(\mathrm{ddt}_{\mathrm{ap}}, 1 \mathrm{H}\right.$, $J_{l}=17,1 \mathrm{~Hz}, J_{2}=10,2 \mathrm{~Hz}$ e $\left.J_{3}=6,7 \mathrm{~Hz}\right) ; 6,65-6,73(\mathrm{~m}, 2 \mathrm{H}) ; 6,92(\mathrm{~d}$, $1 \mathrm{H}, J=7,9 \mathrm{~Hz}) ; 7,11(\mathrm{~d}, 2 \mathrm{H}, J=8,4 \mathrm{~Hz}) ; 7,48(\mathrm{~d}, 2 \mathrm{H}, J=8,4 \mathrm{~Hz}) ; 7,54$ (s, $1 \mathrm{H}) . \mathrm{RMN}^{13} \mathrm{C}\left(75 \mathrm{MHz}, \mathrm{CDCl}_{3}\right) \delta: 39,8 ; 53,4 ; 55,7 ; 63,4 ; 112,2$; 114,$6 ; 115,7 ; 120,4 ; 122,7 ; 122,8 ; 129,6 ; 132,2 ; 133,4 ; 133,9 ; 137,4$; 145,$1 ; 145,7 ; 149,5$. HRMS $\left(\mathrm{M}+\mathrm{H}^{+}\right)$: calculado para $\mathrm{C}_{20} \mathrm{H}_{21} \mathrm{BrN}_{3} \mathrm{O}_{2}$ : 414,08171; encontrado: 414,08072. HRMS $\left(\mathrm{M}+\mathrm{Na}^{+}\right)$: calculado para $\mathrm{C}_{20} \mathrm{H}_{20} \mathrm{BrN}_{3} \mathrm{NaO}_{2}$ : 436,06366; encontrado: 436,06058.

Dados para 4-((4-alil-2-metoxifenoxi)metil)-1-(4-iodobenzil)-1H-1,2,3-triazol (6e): Sólido branco, purificado por cromatografia em coluna eluída com hexano-acetato de etila-diclorometano (3:1:3 v/v), P.f. $=129,2-131,1^{\circ} \mathrm{C}, \mathrm{R}_{\mathrm{f}}=0,37$ (hexano-acetato de etila-diclorometano $3: 1: 3 \mathrm{v} / \mathrm{v}$ ). IV (ATR) $v_{\max } / \mathrm{cm}^{-1}: 3124,3063,2932,1587$, 1512, 1486, 1385, 1258, 1228, 1143, 1017, 1008, 911, 801, 769, 592, 485. RMN ${ }^{1} \mathrm{H}\left(300 \mathrm{MHz}, \mathrm{CDCl}_{3}\right) \delta: 3,32$ (d, 2H, $\left.J=6,6 \mathrm{~Hz}\right)$; 3,82 (s, 3H); 5,02-5,12 (m, 2H); 5,20 (s, 2H); 5,44 (s, 2H); 5,94 (ddt $\mathrm{dap}_{1}, 1 \mathrm{H}, J_{1}=17,0 \mathrm{~Hz}, J_{2}=10,5 \mathrm{~Hz}$ e $\left.J_{3}=6,6 \mathrm{~Hz}\right) ; 6,65-6,73(\mathrm{~m}$, $2 \mathrm{H}) ; 6,92$ (d, $1 \mathrm{H}, J=7,9 \mathrm{~Hz}) ; 6,98$ (d, 2H, $J=8,3 \mathrm{~Hz}) ; 7,54$ (s, 1H); $7,69$ (d, $2 \mathrm{H}, J=8,3 \mathrm{~Hz}) . \mathrm{RMN}{ }^{13} \mathrm{C}\left(75 \mathrm{MHz}, \mathrm{CDCl}_{3}\right) \delta: 39,8 ; 53,5$; 55,$7 ; 63,4 ; 94,5 ; 112,2 ; 114,6 ; 115,7 ; 120,4 ; 122,7 ; 129,8 ; 133,9$; 134,$1 ; 137,4 ; 138,2 ; 145,1 ; 145,8 ; 149,5$. HRMS $\left(\mathrm{M}+\mathrm{H}^{+}\right)$: calculado para $\mathrm{C}_{20} \mathrm{H}_{21} \mathrm{IN}_{3} \mathrm{O}_{2}$ : 462,06784; encontrado: 462,06711. HRMS $\left(\mathrm{M}+\mathrm{Na}^{+}\right)$: calculado para $\mathrm{C}_{20} \mathrm{H}_{20} \mathrm{IN}_{3} \mathrm{NaO}_{2}, 484,04979$; encontrado: 484,04898.
Dados para 4-((4-alil-2-metoxifenoxi)metil)-1-(4-nitrobenzil)-1H-1,2,3-triazol (6f): Sólido branco, purificado por cromatografia em coluna eluída com hexano-acetato de etila-diclorometano (3:1:3 v/v), P.f. $=120,9-121,9{ }^{\circ} \mathrm{C}, \mathrm{R}_{\mathrm{f}}=0,14$ (hexano-acetato de etila-diclorometano 3:1:3 v/v). IV (ATR) $v_{\max } / \mathrm{cm}^{-1}: 3063,3005$, 2950, 2917, 1591, 1516, 1350, 1258, 1230, 1140, 1019, 835, 720. $\mathrm{RMN}{ }^{1} \mathrm{H}\left(300 \mathrm{MHz}, \mathrm{CDCl}_{3}\right) \delta: 3,32(\mathrm{~d}, 2 \mathrm{H}, J=6,7 \mathrm{~Hz}) ; 3,81(\mathrm{~s}$, $3 \mathrm{H}) ; 5,01-5,12(\mathrm{~m}, 2 \mathrm{H}) ; 5,25(\mathrm{~s}, 2 \mathrm{H}) ; 5,62(\mathrm{~s}, 2 \mathrm{H}) ; 5,93\left(\mathrm{ddt}_{\mathrm{ap}}, 1 \mathrm{H}\right.$, $J_{I}=17,0 \mathrm{~Hz}, J_{2}=10,3 \mathrm{~Hz}$ e $\left.J_{3}=6,7 \mathrm{~Hz}\right) ; 6,65-6,73(\mathrm{~m}, 2 \mathrm{H}) ; 6,92$ $(\mathrm{d}, 1 \mathrm{H}, J=7,9 \mathrm{~Hz}) ; 7,37(\mathrm{~d}, 2 \mathrm{H}, J=8,6 \mathrm{~Hz}) ; 7,63(\mathrm{~s}, 1 \mathrm{H}) ; 8,20$ $(\mathrm{d}, 2 \mathrm{H}, J=8,6 \mathrm{~Hz})$. RMN ${ }^{13} \mathrm{C}\left(75 \mathrm{MHz}, \mathrm{CDCl}_{3}\right) \delta: 39,7 ; 53,0$; 55,$7 ; 63,3 ; 112,3 ; 114,5 ; 115,7 ; 120,4 ; 123,0 ; 124,2 ; 128,5 ; 134,0$; 137,$4 ; 141,5 ; 145,4 ; 145,7 ; 148,0 ; 149,5$. HRMS $\left(\mathrm{M}+\mathrm{H}^{+}\right)$: calculado para $\mathrm{C}_{20} \mathrm{H}_{21} \mathrm{~N}_{4} \mathrm{O}_{4}$ : 381,15628; encontrado: 381,15533. HRMS $\left(\mathrm{M}+\mathrm{Na}^{+}\right)$: calculado para $\mathrm{C}_{20} \mathrm{H}_{20} \mathrm{~N}_{4} \mathrm{NaO}_{4}$ : 403,13822; encontrado: 403,13715.

Dados para 4-((4-alil-2-metoxifenoxi)metil)-1-(4-metoxibenzil)-1H-1,2,3-triazol (6g): Sólido branco, purificado por cromatografia em coluna eluída com hexano-acetato de etila-diclorometano (3:1:3 $\mathrm{v} / \mathrm{v}$ ), P.f. $=92,4-93,1^{\circ} \mathrm{C}, \mathrm{R}_{\mathrm{f}}=0,36$ (hexano-acetato de etila-diclorometano $3: 1: 3 \mathrm{v} / \mathrm{v})$. IV (ATR) $v_{\text {max }} / \mathrm{cm}^{-1}: 3068,3005,2936,2832,1613$, 1587, 1512, 1463, 1251, 1227, 1142, 1016, 903, 805, 774, 702, 548. RMN ${ }^{1} \mathrm{H}\left(300 \mathrm{MHz}, \mathrm{CDCl}_{3}\right) \delta: 3,31(\mathrm{~d}, 2 \mathrm{H}, J=6,7 \mathrm{~Hz}) ; 3,80(\mathrm{~s}, 3 \mathrm{H})$; $3,81(\mathrm{~s}, 3 \mathrm{H}) ; 5,01-5,12(\mathrm{~m}, 2 \mathrm{H}) ; 5,21(\mathrm{~s}, 2 \mathrm{H}) ; 5,43(\mathrm{~s}, 2 \mathrm{H}) ; 5,94\left(\mathrm{ddt}_{\mathrm{ap}}\right.$, $1 \mathrm{H}, J_{1}=16,9 \mathrm{~Hz}, J_{2}=10,2 \mathrm{~Hz}$ e $\left.J_{3}=6,7 \mathrm{~Hz}\right) ; 6,65-6,73(\mathrm{~m}, 2 \mathrm{H}) ; 6,88$ $(\mathrm{d}, 2 \mathrm{H}, J=8,6 \mathrm{~Hz}) ; 6,94(\mathrm{~d}, 1 \mathrm{H}, J=7,8 \mathrm{~Hz}) ; 7,21(\mathrm{~d}, 2 \mathrm{H}, J=8,6 \mathrm{~Hz})$; $7,51$ (s, $1 \mathrm{H}) . \mathrm{RMN}{ }^{13} \mathrm{C}\left(75 \mathrm{MHz}, \mathrm{CDCl}_{3}\right) \delta: 39,8 ; 53,7 ; 55,3 ; 55,7$; 63,$5 ; 112,2 ; 114,4 ; 114,5 ; 115,6 ; 120,4 ; 122,5 ; 126,4 ; 129,7 ; 133,7$; 137,$4 ; 144,7145,9 ; 149,5 ; 159,9$. HRMS $\left(\mathrm{M}+\mathrm{H}^{+}\right)$: calculado para $\mathrm{C}_{21} \mathrm{H}_{24} \mathrm{~N}_{3} \mathrm{O}_{3}$ : 366,18177; encontrado: 366,18069. HRMS (M+Na+): calculado para $\mathrm{C}_{21} \mathrm{H}_{23} \mathrm{~N}_{3} \mathrm{NaO}_{3}$ : 388,16371; encontrado: 388,16229 .

Dados para 4-((4-alil-2-metoxifenoxi)metil)-1-(4-(trifluorometoxi)benzil)-1H-1,2,3-triazol (6h): Sólido branco, purificado por cromatografia em coluna eluída com hexano-acetato de etila-diclorometano $(3: 1: 3 \mathrm{v} / \mathrm{v})$, P.f. $=126,4-127,3{ }^{\circ} \mathrm{C}, \mathrm{R}_{\mathrm{f}}=0,49$ (hexano-acetato de etila-diclorometano $3: 1: 3 \mathrm{v} / \mathrm{v}$ ). IV (ATR) $v_{\max } / \mathrm{cm}^{-1}: 3065,3004$, 2978, 2941, 1589, 1510, 1467, 1387, 1256, 1218, 1142, 1017, 915, 804. RMN ${ }^{1} \mathrm{H}\left(300 \mathrm{MHz}, \mathrm{CDCl}_{3}\right) \delta: 3,32(\mathrm{~d}, 2 \mathrm{H}, J=6,7 \mathrm{~Hz}) ; 3,81$ $(\mathrm{s}, 3 \mathrm{H}) ; 5,02-5,12(\mathrm{~m}, 2 \mathrm{H}) ; 5,24(\mathrm{~s}, 2 \mathrm{H}) ; 5,51(\mathrm{~s}, 2 \mathrm{H}) ; 5,94\left(\mathrm{ddt}_{\mathrm{ap}}, 1 \mathrm{H}\right.$, $J_{1}=17,0 \mathrm{~Hz}, J_{2}=10,2 \mathrm{~Hz}$ e $\left.J_{3}=6,7 \mathrm{~Hz}\right) ; 6,65-6,73(\mathrm{~m}, 2 \mathrm{H}) ; 6,93(\mathrm{~d}$, $1 \mathrm{H}, J=7,9 \mathrm{~Hz}) ; 7,20(\mathrm{~d}, 2 \mathrm{H}, J=8,5 \mathrm{~Hz}) ; 7,28(\mathrm{~d}, 2 \mathrm{H}, J=8,5 \mathrm{~Hz})$; 7,57 (s, $1 \mathrm{H}) . \mathrm{RMN}{ }^{13} \mathrm{C}\left(75 \mathrm{MHz}, \mathrm{CDCl}_{3}\right) \delta: 39,7 ; 53,2 ; 55,8 ; 63,4$; $112,2 ; 114,5 ; 115,8 ; 120,3$ (q, $J=256,3 \mathrm{~Hz}) ; 120,4 ; 121,5 ; 122,7$; 129,$5 ; 133,2 ; 133,9 ; 137,4 ; 145,1 ; 145,8 ; 149,3-149,2$ (m); 149,5. HRMS $\left(\mathrm{M}+\mathrm{H}^{+}\right)$: calculado para $\mathrm{C}_{21} \mathrm{H}_{21} \mathrm{~F}_{3} \mathrm{~N}_{3} \mathrm{O}_{3}$ : 420,15350; encontrado: 420,15250. HRMS $\left(\mathrm{M}+\mathrm{Na}^{+}\right)$: calculado para $\mathrm{C}_{21} \mathrm{H}_{20} \mathrm{~F}_{3} \mathrm{~N}_{3} \mathrm{NaO}_{3}$ : 442,13545; encontrado: 442,13422.

Dados para 4-((4-alil-2-metoxifenoxi)metil)-1-(4-(trifluorometil) benzil)-1H-1,2,3-triazol (6i): Sólido branco, purificado por cromatografia em coluna eluída com hexano-acetato de etila-diclorometano $(3: 1: 3 \mathrm{v} / \mathrm{v})$, P.f. $=142,7-143,0^{\circ} \mathrm{C}, \mathrm{R}_{\mathrm{f}}=0,43$ (hexano-acetato de etila-diclorometano $3: 1: 3 \mathrm{v} / \mathrm{v}$ ). IV (ATR) $v_{\max } / \mathrm{cm}^{-1}: 3060,3003,2977$, 2944, 1589, 1515, 1422, 1328, 1229, 1142, 1117, 1017, 913, 799, 624. $\mathrm{RMN}{ }^{1} \mathrm{H}\left(300 \mathrm{MHz}, \mathrm{CDCl}_{3}\right) \delta: 3,32(\mathrm{~d}, 2 \mathrm{H}, J=6,7 \mathrm{~Hz}) ; 3,81$ (s, 3H); 5,02-5,12 (m, 2H); 5,25 (s, 2H); 5,57 (s, 2H); 5,94 (ddt ${ }_{\mathrm{ap}}, 1 \mathrm{H}$, $J_{I}=17,0 \mathrm{~Hz}, J_{2}=10,2 \mathrm{~Hz}$ e $\left.J_{3}=6,7 \mathrm{~Hz}\right) ; 6,65-6,73(\mathrm{~m}, 2 \mathrm{H}) ; 6,93(\mathrm{~d}$, $1 \mathrm{H}, J=7,8 \mathrm{~Hz}) ; 7,34(\mathrm{~d}, 2 \mathrm{H}, J=8,1 \mathrm{~Hz}) ; 7,58(\mathrm{sl}, 1 \mathrm{H}) ; 7,61(\mathrm{~d}, 2 \mathrm{H}$, $J=8,1 \mathrm{~Hz})$. RMN ${ }^{13} \mathrm{C}\left(75 \mathrm{MHz}, \mathrm{CDCl}_{3}\right) \delta: 39,7 ; 53,4 ; 55,8 ; 63,4$; $112,2 ; 114,6 ; 115,7 ; 120,4 ; 122,8 ; 123,7$ (q, $J=270,8 \mathrm{~Hz}) ; 126,0$ $(\mathrm{q}, J=3,8 \mathrm{~Hz}) ; 128,1 ; 131,0(\mathrm{q}, J=32,5 \mathrm{~Hz}) ; 133,9 ; 137,4 ; 138,4$; 145,$2 ; 145,7 ; 149,5$. HRMS $\left(\mathrm{M}+\mathrm{H}^{+}\right)$: calculado para $\mathrm{C}_{21} \mathrm{H}_{21} \mathrm{~F}_{3} \mathrm{~N}_{3} \mathrm{O}_{2}$ : 404,15859; encontrado: 404,15771. HRMS (M+Na+): calculado para $\mathrm{C}_{21} \mathrm{H}_{20} \mathrm{~F}_{3} \mathrm{~N}_{3} \mathrm{NaO}_{2}$ : 426,14053; encontrado: 426,13928. 
Dados para 4-((4-alil-2-metoxifenoxi)metil)-1-(2,5-diclorobenzil)-1H-1,2,3-triazol (6j): Sólido branco, purificado por cromatografia em coluna eluída com hexano-acetato de etila-diclorometano (3:1:3 v/v), P.f. $=87,9-88,4^{\circ} \mathrm{C}, \mathrm{R}_{\mathrm{f}}=0,59$ (hexano-acetato de etila-diclorometano $3: 1: 3 \mathrm{v} / \mathrm{v}$ ). IV (ATR) $v_{\max } / \mathrm{cm}^{-1}: 3143,3008,2962,2871,1587$ $1508,1460,1391,1256,1213,1137,1030,1003,905,821,797,645$, 560. $\mathrm{RMN}{ }^{1} \mathrm{H}\left(300 \mathrm{MHz}, \mathrm{CDCl}_{3}\right) \delta: 3,32(\mathrm{~d}, 2 \mathrm{H}, J=6,7 \mathrm{~Hz}) ; 3,83(\mathrm{~s}$, $3 \mathrm{H}) ; 5,01-5,12(\mathrm{~m}, 2 \mathrm{H}) ; 5,23(\mathrm{~s}, 2 \mathrm{H}) ; 5,60(\mathrm{~s}, 2 \mathrm{H}) ; 5,94\left(\mathrm{ddt}_{\mathrm{ap}}, 1 \mathrm{H}\right.$, $J_{1}=16,9 \mathrm{~Hz}, J_{2}=9,9 \mathrm{~Hz}$ e $\left.J_{3}=6,7 \mathrm{~Hz}\right) ; 6,68-6,71(\mathrm{~m}, 2 \mathrm{H}) ; 6,93(\mathrm{~d}$, $1 \mathrm{H}, J=7,8 \mathrm{~Hz}) ; 7,12(\mathrm{~d}, 1 \mathrm{H}, J=2,3 \mathrm{~Hz}) ; 7,27\left(\mathrm{dd}, 1 \mathrm{H}, J_{1}=8,6 \mathrm{~Hz}\right.$ e $\left.J_{2}=2,3 \mathrm{~Hz}\right) ; 7,35(\mathrm{~d}, 1 \mathrm{H}, J=8,6 \mathrm{~Hz}) ; 7,67(\mathrm{~s}, 1 \mathrm{H}) . \mathrm{RMN}{ }^{13} \mathrm{C}$ $\left(75 \mathrm{MHz}, \mathrm{CDCl}_{3}\right) \delta$ : 39,8; 50,9; 55,8; 63,5; 112,3; 114,7; 115,7; 120,$5 ; 123,1 ; 130,0 ; 130,2 ; 130,9131,5 ; 133,5 ; 133,97 ; 133,99 ; 137,4$; 145,$1 ; 145,7 ; 149,5$. HRMS $\left(\mathrm{M}+\mathrm{H}^{+}\right)$: calculado para $\mathrm{C}_{20} \mathrm{H}_{20} \mathrm{Cl}_{2} \mathrm{~N}_{3} \mathrm{O}_{2}$ : 404,09326; encontrado: 404,09232. HRMS $\left(\mathrm{M}+\mathrm{Na}^{+}\right)$: calculado para $\mathrm{C}_{20} \mathrm{H}_{19} \mathrm{Cl}_{2} \mathrm{~N}_{3} \mathrm{NaO}_{2}$ : 426,07520; encontrado: 426,07388.

Dados para 4-((4-alil-2-metoxifenoxi)metil)-1-(3,4-difluorobenzil)-1H-1,2,3-triazol (6k): Sólido branco, purificado por cromatografia em coluna eluída com hexano-acetato de etila-diclorometano $(3: 1: 3 \mathrm{v} / \mathrm{v})$, P.f. $=104,9-105,2^{\circ} \mathrm{C}, \mathrm{R}_{\mathrm{f}}=0,30$ (hexano-acetato de etila-diclorometano $3: 1: 3 \mathrm{v} / \mathrm{v}$ ). IV (ATR) $v_{\max } / \mathrm{cm}^{-1}: 3065,3003,2939$, 2915, 1589, 1516, 1447, 1287, 1259, 1213, 1142, 1018, 835, 756, 575. $\mathrm{RMN}{ }^{1} \mathrm{H}\left(300 \mathrm{MHz}, \mathrm{CDCl}_{3}\right) \delta: \delta: 3,32(\mathrm{~d}, 2 \mathrm{H}, J=6,7 \mathrm{~Hz}) ; 3,82(\mathrm{~s}$, $3 \mathrm{H}) ; 5,01-5,12(\mathrm{~m}, 2 \mathrm{H}) ; 5,24(\mathrm{~s}, 2 \mathrm{H}) ; 5,45(\mathrm{~s}, 2 \mathrm{H}) ; 5,94\left(\mathrm{ddt}_{\mathrm{ap}}, 1 \mathrm{H}\right.$, $J_{1}=17,0 \mathrm{~Hz}, J_{2}=10,3 \mathrm{~Hz}$ e $\left.J_{3}=6,7 \mathrm{~Hz}\right) ; 6,65-6,73(\mathrm{~m}, 2 \mathrm{H}) ; 6,92(\mathrm{~d}$, $1 \mathrm{H}, J=7,9 \mathrm{~Hz}) ; 6,97-7,19(\mathrm{~m}, 3 \mathrm{H}) ; 7,57(\mathrm{~s}, 1 \mathrm{H}) . \mathrm{RMN}{ }^{13} \mathrm{C}(75 \mathrm{MHz}$, $\left.\mathrm{CDCl}_{3}\right) \delta: 39,7 ; 53,0($ brd, $J=1,0 \mathrm{~Hz}) ; 55,7 ; 63,4 ; 112,2 ; 114,6 ; 115,7$; $117,2(\mathrm{~d}, J=18,0 \mathrm{~Hz}) ; 117,9(\mathrm{~d}, J=18,0 \mathrm{~Hz}) ; 120,4 ; 122,7 ; 124,2$ (dd, $J_{l}=6,8 \mathrm{~Hz}$ e $\left.J_{2}=3,8 \mathrm{~Hz}\right) ; 131,4-131,5$ (m); 133,9; 137,4; 145,2; 145,$6 ; 149,5 ; 148,7-148,9(\mathrm{~m}) ; 152,0-152,2(\mathrm{~m})$. HRMS $\left(\mathrm{M}+\mathrm{H}^{+}\right)$: calculado para $\mathrm{C}_{20} \mathrm{H}_{20} \mathrm{~F}_{2} \mathrm{~N}_{3} \mathrm{O}_{2}$ : 372,15236; encontrado: 372,15137. HRMS $\left(\mathrm{M}+\mathrm{Na}^{+}\right)$: calculado para $\mathrm{C}_{20} \mathrm{H}_{19} \mathrm{~F}_{2} \mathrm{~N}_{3} \mathrm{NaO}_{2}$ : 394,13430 ; encontrado: 394,13312 .

Dados para 4-((4-alil-2-metoxifenoxi)metil)-1-(4-metilbenzil)-1H-1,2,3-triazol (6l): Sólido branco, purificado por cromatografia em coluna eluída com hexano-acetato de etila-diclorometano $(3: 1: 3 \mathrm{v} / \mathrm{v})$, P.f. $=81,4-82,3^{\circ} \mathrm{C}, \mathrm{R}_{\mathrm{f}}=0,66$ (hexano-acetato de etila-diclorometano $3: 1: 3 \mathrm{v} / \mathrm{v})$. IV (ATR) $v_{\max } / \mathrm{cm}^{-1}: 3064,3003,2917,1588,1513,1446$, $1257,1229,1142,1017,907,803,756 . \mathrm{RMN}^{1} \mathrm{H}\left(300 \mathrm{MHz}, \mathrm{CDCl}_{3}\right) \delta$ : $2,34$ (s, 3H); 3,32 (d, 2H, $J=6,6 \mathrm{~Hz}) ; 3,81$ (s, 3H); 5,01-5,12 (m, 2H); $5,22(\mathrm{~s}, 2 \mathrm{H}) ; 5,45(\mathrm{~s}, 2 \mathrm{H}) ; 5,94\left(\mathrm{ddt}_{\mathrm{ap}}, 1 \mathrm{H}, J_{1}=16,9 \mathrm{~Hz}, J_{2}=10,2 \mathrm{~Hz}\right.$ e $\left.J_{3}=6,6 \mathrm{~Hz}\right) ; 6,65-6,73(\mathrm{~m}, 2 \mathrm{H}) ; 6,93(\mathrm{~d}, 1 \mathrm{H}, J=7,9 \mathrm{~Hz}) ; 7,15(\mathrm{~s}, 4 \mathrm{H})$; $7,52(\mathrm{~s}, 1 \mathrm{H}) . \mathrm{RMN}{ }^{13} \mathrm{C}\left(75 \mathrm{MHz}, \mathrm{CDCl}_{3}\right) \delta: 21,1 ; 39,8 ; 53,9 ; 55,7$; 63,$5 ; 112,2 ; 114,6 ; 115,6 ; 120,4 ; 122,6 ; 128,1 ; 129,7 ; 131,4 ; 133,8$; 137,$5 ; 138,6 ; 144,7 ; 145,9 ; 149,5$. HRMS $\left(\mathrm{M}+\mathrm{H}^{+}\right)$: calculado para $\mathrm{C}_{21} \mathrm{H}_{24} \mathrm{~N}_{3} \mathrm{O}_{2}$ : 350,18685; encontrado: 350,18582. HRMS (M+Na+): calculado para $\mathrm{C}_{21} \mathrm{H}_{23} \mathrm{~N}_{3} \mathrm{NaO}_{2}$ : 372,16880; encontrado: 372,16776.

Dados para 4-((4-alil-2-metoxifenoxi)metil)-1-(2-bromobenzil)-1H-1,2,3-triazol (6m): Sólido branco, purificado por cromatografia em coluna eluída com hexano-acetato de etila-diclorometano $(3: 1: 3 \mathrm{v} / \mathrm{v})$, P.f. $=67,5-68,6^{\circ} \mathrm{C}, \mathrm{R}_{\mathrm{f}}=0,70$ (hexano-acetato de etila-diclorometano $3: 1: 3 \mathrm{v} / \mathrm{v}$ ). IV (ATR) $v_{\max } / \mathrm{cm}^{-1}: 3141,3079,3003,2944,2873,1587$, 1509, 1421, 1389, 1254, 1213, 1134, 1050, 1026, 1000, 920, 759, 649, 596. $\mathrm{RMN}{ }^{1} \mathrm{H}\left(300 \mathrm{MHz}, \mathrm{CDCl}_{3}\right) \delta: 3,32(\mathrm{~d}, 2 \mathrm{H}, J=6,6 \mathrm{~Hz}) ; 3,82$ (s, 3H); 5,00-5,11 (m, 2H); 5,25 (s, 2H); 5,64 (s, 2H); 5,94 (ddt $\mathrm{ap}_{\text {, }} 1 \mathrm{H}$, $J_{I}=16,9 \mathrm{~Hz}, J_{2}=10,2 \mathrm{~Hz}$ e $\left.J_{3}=6,6 \mathrm{~Hz}\right) ; 6,65-6,73(\mathrm{~m}, 2 \mathrm{H}) ; 6,95(\mathrm{~d}$, $1 \mathrm{H}, J=7,8 \mathrm{~Hz}) ; 7,12\left(\mathrm{dd}, 1 \mathrm{H}, J_{1}=7,4 \mathrm{~Hz}\right.$ e $\left.J_{2}=1,7 \mathrm{~Hz}\right) ; 7,17-7,33(\mathrm{~m}$, $2 \mathrm{H}) ; 7,60\left(\mathrm{dd}, 1 \mathrm{H}, J_{1}=7,8 \mathrm{~Hz}\right.$ e $\left.J_{2}=1,2 \mathrm{~Hz}\right) ; 7,66(\mathrm{~s}, 1 \mathrm{H}) . \mathrm{RMN}{ }^{13} \mathrm{C}$ $\left(75 \mathrm{MHz}, \mathrm{CDCl}_{3}\right) \delta: 39,7 ; 53,7 ; 55,7 ; 63,5 ; 112,2 ; 114,7 ; 115,6 ; 120,4$; 123,$1 ; 123,4 ; 128,1 ; 130,30 ; 130,33 ; 133,1 ; 133,8 ; 134,0 ; 137,4$ 144,$7 ; 145,7 ; 149,5$. HRMS $\left(\mathrm{M}+\mathrm{H}^{+}\right)$: calculado para $\mathrm{C}_{20} \mathrm{H}_{21} \mathrm{BrN}_{3} \mathrm{O}_{2}$ : 414,08171; encontrado: 414,08096. HRMS $\left(\mathrm{M}+\mathrm{Na}^{+}\right)$: calculado para $\mathrm{C}_{20} \mathrm{H}_{20} \mathrm{BrN}_{3} \mathrm{NaO}_{2}$ : 436,06366; encontrado: 436,06290.
Dados para 4-((4-alil-2-metoxifenoxi)metil)-1-(3-bromobenzil)-1H-1,2,3-triazol (6n): Sólido branco, purificado por cromatografia em coluna eluída com hexano-acetato de etila-diclorometano $(3: 1: 3 \mathrm{v} / \mathrm{v})$, P.f. $=97,5-97,9^{\circ} \mathrm{C}, \mathrm{R}_{\mathrm{f}}=0,70$ (hexano-acetato de etila-diclorometano 3:1:3 v/v). IV (ATR) $v_{\max } / \mathrm{cm}^{-1}: 3065,3011,2936$, 1588, 1512, 1463, 1387, 1226, 1142, 1017, 835, 735, 692. 665. $\mathrm{RMN}{ }^{1} \mathrm{H}\left(300 \mathrm{MHz}, \mathrm{CDCl}_{3}\right) \delta: 3,32(\mathrm{~d}, 2 \mathrm{H}, J=6,6 \mathrm{~Hz}) ; 3,82(\mathrm{~s}$, $3 \mathrm{H}) ; 5,00-5,13(\mathrm{~m}, 2 \mathrm{H}) ; 5,24(\mathrm{~s}, 2 \mathrm{H}) ; 5,47(\mathrm{~s}, 2 \mathrm{H}) ; 5,94\left(\mathrm{ddt}_{\mathrm{ap}}, 1 \mathrm{H}\right.$, $J_{1}=16,9 \mathrm{~Hz}, J_{2}=10,8 \mathrm{~Hz}$ e $\left.J_{3}=6,6 \mathrm{~Hz}\right) ; 6,65-6,73(\mathrm{~m}, 2 \mathrm{H}) ; 6,93$ $(\mathrm{d}, 1 \mathrm{H}, J=7,8 \mathrm{~Hz})$; [7,13-7,26 (m), 7,40 (sl), 7,48 (d $\left.\mathrm{d}_{\mathrm{ap}}, J=7,7 \mathrm{~Hz}\right)$, $4 \mathrm{H}] ; 7,57$ (s, 1H). RMN ${ }^{13} \mathrm{C}\left(75 \mathrm{MHz}, \mathrm{CDCl}_{3}\right) \delta: 39,8 ; 53,3 ; 55,7$; 63,$4 ; 112,2 ; 114,6 ; 115,7 ; 120,5 ; 122,7 ; 123,0 ; 126,6 ; 130,6 ; 131,0$; 131,$9 ; 133,9 ; 136,6 ; 137,4 ; 145,1 ; 145,8 ; 149,5$. HRMS $\left(\mathrm{M}+\mathrm{H}^{+}\right)$: calculado para $\mathrm{C}_{20} \mathrm{H}_{21} \mathrm{BrN}_{3} \mathrm{O}_{2}$ : 414,08171; encontrado: 414,08069. HRMS (M+Na+): calculado para $\mathrm{C}_{20} \mathrm{H}_{20} \mathrm{BrN}_{3} \mathrm{NaO}_{2}$ : 436,06366; encontrado: 436,06265.

Dados para 4-(3-(4-alil-2-metoxifenoxi)propil)-1-benzil-1 $H$ -1,2,3-triazol (7a): Sólido branco, purificado por cromatografia em coluna eluída com hexano-acetato de etila-diclorometano $(3: 1: 3 \mathrm{v} / \mathrm{v})$, P.f. $=71,4-72,6^{\circ} \mathrm{C}, \mathrm{R}_{\mathrm{f}}=0,16$ (hexano-acetato de etila-diclorometano $3: 1: 3 \mathrm{v} / \mathrm{v}$ ). IV (ATR) $v_{\max } / \mathrm{cm}^{-1}: 3059,3007,2958,2830,2876,1588$, 1513, 1461, 1332, 1260, 1230, 1140, 1026, 915, 799, 709, 663. $\mathrm{RMN}{ }^{1} \mathrm{H}\left(300 \mathrm{MHz}, \mathrm{CDCl}_{3}\right.$ ) $\delta: 2,17$ (quint, $2 \mathrm{H}, J=7,0 \mathrm{~Hz}$ ); 2,90 (t, 2H, $J=7,5 \mathrm{~Hz}) ; 3,32(\mathrm{~d}, 2 \mathrm{H}, J=6,7 \mathrm{~Hz}) ; 3,81(\mathrm{~s}, 3 \mathrm{H}) ; 4,01(\mathrm{t}$, $2 \mathrm{H}, J=6,4 \mathrm{~Hz}) ; 5,01-5,11(\mathrm{~m}, 2 \mathrm{H}) ; 5,48(\mathrm{~s}, 2 \mathrm{H}) ; 5,95\left(\mathrm{ddt}_{\mathrm{ap}}, 1 \mathrm{H}\right.$, $J_{l}=16,8 \mathrm{~Hz}, J_{2}=10,2 \mathrm{~Hz}$ e $\left.J_{3}=6,7 \mathrm{~Hz}\right) ; 6,65-6,73(\mathrm{~m}, 2 \mathrm{H}) ; 6,77$ $(\mathrm{d}, 1 \mathrm{H}, J=7,9 \mathrm{~Hz}) ; 7,22-7,37(\mathrm{~m}, 6 \mathrm{H}) . \mathrm{RMN}{ }^{13} \mathrm{C}\left(75 \mathrm{MHz}, \mathrm{CDCl}_{3}\right)$

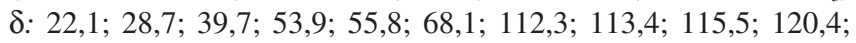
120,$9 ; 127,9 ; 128,5 ; 129,0 ; 132,9 ; 134,8 ; 137,6 ; 146,6 ; 147,7 ; 149,3$. HRMS $\left(\mathrm{M}+\mathrm{H}^{+}\right)$: calculado para $\mathrm{C}_{22} \mathrm{H}_{25} \mathrm{~N}_{3} \mathrm{O}_{2}: 364,20250$; encontrado: 364,20148 . HRMS $\left(\mathrm{M}+\mathrm{Na}^{+}\right)$: calculado para $\mathrm{C}_{22} \mathrm{H}_{25} \mathrm{~N}_{3} \mathrm{NaO}_{2}$ : 386,18445; encontrado: 386,18341.

Dados para 4-(3-(4-alil-2-metoxifenoxi)propil)-1-(4-fluorobenzil)-1H-1,2,3-triazol (7b): Sólido branco, purificado por cromatografia em coluna eluída com hexano-acetato de etila-diclorometano $(3: 1: 3 \mathrm{v} / \mathrm{v})$, P.f. $=96,4-96,8^{\circ} \mathrm{C}, \mathrm{R}_{\mathrm{f}}=0,21$ (hexano-acetato de etila-diclorometano 3:1:3 v/v). IV (ATR) $v_{\max } / \mathrm{cm}^{-1}: 3111,3061,3008$, 2951, 1587, 1511, 1455, 1260, 1222, 1140, 1015, 905, 798, 772, 654. RMN ${ }^{1} \mathrm{H}\left(300 \mathrm{MHz}, \mathrm{CDCl}_{3}\right) \delta: 2,17$ (quint, $2 \mathrm{H}, J=6,9 \mathrm{~Hz}$ ); $2,90$ (t, 2H, $J=7,5 \mathrm{~Hz}) ; 3,32$ (d, 2H, $J=6,7 \mathrm{~Hz}) ; 3,81$ (s, 3H); 4,01 (t, 2H, $J=6,4 \mathrm{~Hz}) ; 5,00-5,13(\mathrm{~m}, 2 \mathrm{H}) ; 5,44(\mathrm{~s}, 2 \mathrm{H}) ; 5,95\left(\mathrm{ddt}_{\mathrm{ap}}, 1 \mathrm{H}\right.$, $J_{1}=16,8 \mathrm{~Hz}, J_{2}=10,8 \mathrm{~Hz}$ e $\left.J_{3}=6,7 \mathrm{~Hz}\right) ; 6,65-6,73(\mathrm{~m}, 2 \mathrm{H}) ; 6,77(\mathrm{~d}$, $1 \mathrm{H}, J=7,9 \mathrm{~Hz}) ; 7,03(\mathrm{t}, 2 \mathrm{H}, J=8,6 \mathrm{~Hz}) ; 7,16-7,30(\mathrm{~m}, 3 \mathrm{H}) . \mathrm{RMN}{ }^{13} \mathrm{C}$ $\left(75 \mathrm{MHz}, \mathrm{CDCl}_{3}\right) \delta: 22,1 ; 28,7 ; 39,7 ; 53,2 ; 55,8 ; 68,0 ; 112,3 ; 113,4$; $115,6 ; 116,0(\mathrm{~d}, J=21,8 \mathrm{~Hz}) ; 120,4 ; 120,8 ; 129,7$ (d, $J=8,3 \mathrm{~Hz})$; $130,7(\mathrm{~d}, J=3,0 \mathrm{~Hz}) ; 132,9 ; 137,6 ; 146,6 ; 147,8 ; 149,3 ; 162,7$ $(\mathrm{d}, J=246,8 \mathrm{~Hz})$. HRMS $\left(\mathrm{M}+\mathrm{H}^{+}\right)$: calculado para $\mathrm{C}_{22} \mathrm{H}_{25} \mathrm{FrN}_{3} \mathrm{O}_{2}$ : 382,19308; encontrado: 382,19211. HRMS $\left(\mathrm{M}+\mathrm{Na}^{+}\right)$: calculado para $\mathrm{C}_{22} \mathrm{H}_{24} \mathrm{FN}_{3} \mathrm{NaO}_{2}$ : 404,17502; encontrado: 404,17404.

Dados para 4-(3-(4-alil-2-metoxifenoxi)propil)-1-(4-clorobenzil)-1H-1,2,3-triazol (7c): Sólido branco, purificado por cromatografia em coluna eluída com hexano-acetato de etila-diclorometano (3:1:3 $\mathrm{v} / \mathrm{v}$ ), P.f. $=81,8-82,2^{\circ} \mathrm{C}, \mathrm{R}_{\mathrm{f}}=0,46$ (hexano-acetato de etila-diclorometano $3: 1: 3 \mathrm{v} / \mathrm{v}$ ). IV (ATR) $v_{\max } / \mathrm{cm}^{-1}: 3111,3063,3000,2937$, 2856, 1589, 1514, 1446, 1259, 1227, 1140, 1015, 909, 799, 776, 739, 641. $\mathrm{RMN}{ }^{1} \mathrm{H}\left(300 \mathrm{MHz}, \mathrm{CDCl}_{3}\right) \delta: 2,17$ (quint, $2 \mathrm{H}, J=6,9 \mathrm{~Hz}$ ); $2,90$ (t, 2H, $J=7,5 \mathrm{~Hz}) ; 3,32(\mathrm{~d}, 2 \mathrm{H}, J=6,7 \mathrm{~Hz}) ; 3,82(\mathrm{~s}, 3 \mathrm{H}) ; 4,01$ (t, 2H, $J=6,4 \mathrm{~Hz}) ; 5,00-5,11(\mathrm{~m}, 2 \mathrm{H}) ; 5,44(\mathrm{~s}, 2 \mathrm{H}) ; 5,95\left(\mathrm{ddt}_{\mathrm{ap}}, 1 \mathrm{H}\right.$, $J_{I}=17,0 \mathrm{~Hz}, J_{2}=10,2 \mathrm{~Hz}$ e $\left.J_{3}=6,7 \mathrm{~Hz}\right) ; 6,65-6,73(\mathrm{~m}, 2 \mathrm{H}) ; 6,77(\mathrm{~d}$, $1 \mathrm{H}, J=7,9 \mathrm{~Hz}) ; 7,16(\mathrm{~d}, 2 \mathrm{H}, J=8,5 \mathrm{~Hz}) ; 7,24(\mathrm{~s}, 1 \mathrm{H}) ; 7,32(\mathrm{~d}, 2 \mathrm{H}$, $J=8,5 \mathrm{~Hz}) . \mathrm{RMN}{ }^{13} \mathrm{C}\left(75 \mathrm{MHz}, \mathrm{CDCl}_{3}\right) \delta: 22,1 ; 28,7 ; 39,7 ; 53,2$; 55,$8 ; 68,0 ; 112,3 ; 113,4 ; 115,6 ; 120,4 ; 120,9 ; 129,2 ; 132,9 ; 133,3$; 134,$6 ; 137,6 ; 146,6 ; 147,9 ; 149,3^{*}$. HRMS $\left(\mathrm{M}+\mathrm{H}^{+}\right)$: calculado para 
$\mathrm{C}_{21} \mathrm{H}_{25} \mathrm{ClN}_{3} \mathrm{O}_{2}: 398,16353$; encontrado: 398,16272 . HRMS $\left(\mathrm{M}+\mathrm{Na}^{+}\right)$: calculado para $\mathrm{C}_{22} \mathrm{H}_{24} \mathrm{ClN}_{3} \mathrm{NaO}_{2}$ : 420,14547; encontrado: 420,14474.

*Para este composto, houve a sobreposição de sinais de átomos de carbono.

Dados para 4-(3-(4-alil-2-metoxifenoxi)propil)-1-(4-bromobenzil)-1H-1,2,3-triazol (7d): Sólido branco, purificado por cromatografia em coluna eluída com hexano-acetato de etila-diclorometano $(3: 1: 3 \mathrm{v} / \mathrm{v})$, P.f. $=89,4-90,3{ }^{\circ} \mathrm{C}, \mathrm{R}_{\mathrm{f}}=0,46$ (hexano-acetato de etila-diclorometano 3:1:3 v/v). IV (ATR) $v_{\max } / \mathrm{cm}^{-1}: 3110,3063,2999$, 2950, 1588, 1514, 1459, 1420, 1260, 1227, 1141, 1013, 908, 797, 733, 489. $\delta: 2,17$ (quint, $2 \mathrm{H}, J=6,9 \mathrm{~Hz}$ ); $2,90(\mathrm{t}, 2 \mathrm{H}, J=7,5 \mathrm{~Hz}$ ); $3,32$ (d, 2H, $J=6,7 \mathrm{~Hz}) ; 3,82(\mathrm{~s}, 3 \mathrm{H})$; 4,01 (t, 2H, $J=6,3 \mathrm{~Hz})$; 5,03$5,13(\mathrm{~m}, 2 \mathrm{H}) ; 5,43(\mathrm{~s}, 2 \mathrm{H}) ; 5,95\left(\mathrm{ddt}_{\mathrm{ap}}, 1 \mathrm{H}, J_{1}=16,8 \mathrm{~Hz}, J_{2}=10,2 \mathrm{~Hz}\right.$ e $\left.J_{3}=6,7 \mathrm{~Hz}\right) ; 6,65-6,73(\mathrm{~m}, 2 \mathrm{H}) ; 6,77(\mathrm{~d}, 1 \mathrm{H}, J=7,9 \mathrm{~Hz}) ; 7,10(\mathrm{~d}$, $2 \mathrm{H}, J=8,4 \mathrm{~Hz}) ; 7,24$ (s, $1 \mathrm{H}) ; 7,47$ (d, $2 \mathrm{H}, J=8,4 \mathrm{~Hz})$. RMN ${ }^{13} \mathrm{C}$ $\left(75 \mathrm{MHz}, \mathrm{CDCl}_{3}\right.$ ) $\delta: 22,1 ; 28,7 ; 39,7 ; 53,2 ; 55,8 ; 68,0 ; 112,3 ; 113,4$; 115,$6 ; 120,4 ; 120,9 ; 122,7 ; 129,5 ; 132,2 ; 133,0 ; 133,9 ; 137,6$; 146,$6 ; 147,9 ; 149,4$. HRMS $\left(\mathrm{M}+\mathrm{H}^{+}\right)$: calculado para $\mathrm{C}_{22} \mathrm{H}_{25} \mathrm{BrN}_{3} \mathrm{O}_{2}$ : 442,11301; encontrado: 442,11227. HRMS $\left(\mathrm{M}+\mathrm{Na}^{+}\right)$: calculado para $\mathrm{C}_{22} \mathrm{H}_{24} \mathrm{BrN}_{3} \mathrm{NaO}_{2}$ : 464,09496; encontrado: 464,09402.

Dados para 4-(3-(4-alil-2-metoxifenoxi)propil)-1-(4-iodobenzil)-1H-1,2,3-triazol (7e): Sólido branco, purificado por cromatografia em coluna eluída com hexano-acetato de etila-diclorometano (3:1:3 v/v), P.f. $=98,3-99,2^{\circ} \mathrm{C}, \mathrm{R}_{\mathrm{f}}=0,45$ (hexano-acetato de etila-diclorometano $3: 1: 3 \mathrm{v} / \mathrm{v})$. IV (ATR) $v_{\max } / \mathrm{cm}^{-1}: 3110,3062,2999$, 2951, 1588, 1513, 1461, 1419, 1260, 1227, 1140, 1009, 910, 797, 731, 655, 484. RMN ${ }^{1} \mathrm{H}\left(300 \mathrm{MHz}, \mathrm{CDCl}_{3}\right) \delta: 2,17$ (quint, $2 \mathrm{H}, J=$ $6,9 \mathrm{~Hz}) ; 2,90(\mathrm{t}, 2 \mathrm{H}, J=7,5 \mathrm{~Hz}) ; 3,33(\mathrm{~d}, 2 \mathrm{H}, J=6,6 \mathrm{~Hz}) ; 3,82(\mathrm{~s}$, $3 \mathrm{H}) ; 4,01(\mathrm{t}, 2 \mathrm{H}, J=6,3 \mathrm{~Hz}) ; 5,00-5,12(\mathrm{~m}, 2 \mathrm{H}) ; 5,41$ (s, 2H); 5,95 $\left(\mathrm{ddt}_{\mathrm{ap}}, 1 \mathrm{H}, J_{1}=16,8 \mathrm{~Hz}, J_{2}=10,2 \mathrm{~Hz}\right.$ e $\left.J_{3}=6,6 \mathrm{~Hz}\right) ; 6,65-6,73(\mathrm{~m}$, $2 \mathrm{H}) ; 6,77(\mathrm{~d}, 1 \mathrm{H}, J=7,9 \mathrm{~Hz}), 6,96(\mathrm{~d}, 2 \mathrm{H}, J=8,3 \mathrm{~Hz}) ; 7,24(\mathrm{~s}, 1 \mathrm{H})$; $7,68(\mathrm{~d}, 2 \mathrm{H}, J=8,3 \mathrm{~Hz})$. RMN ${ }^{13} \mathrm{C}\left(75 \mathrm{MHz}, \mathrm{CDCl}_{3}\right) \delta: 22,1 ; 28,7$; 39,$7 ; 53,3 ; 55,8 ; 68,0 ; 94,3 ; 112,3 ; 113,4 ; 115,6 ; 120,4 ; 120,9 ; 129,6$; 132,$9 ; 134,5 ; 137,6 ; 138,1 ; 146,6 ; 147,9 ; 149,3$. HRMS $\left(\mathrm{M}+\mathrm{H}^{+}\right)$: calculado para $\mathrm{C}_{22} \mathrm{H}_{25} \mathrm{OIN}_{3} \mathrm{O}_{2}$ : 490,09915; encontrado: 490,09836. HRMS $\left(\mathrm{M}+\mathrm{Na}^{+}\right)$: calculado para for $\mathrm{C}_{22} \mathrm{H}_{24} \mathrm{IN}_{3} \mathrm{NaO}_{2}$ : 512,08109; encontrado: 512,08038.

Dados para 4-(3-(4-alil-2-metoxifenoxi)propil)-1-(4-nitrobenzil)-1H-1,2,3-triazol (7f): Sólido branco, purificado por cromatografia em coluna eluída com hexano-acetato de etila-diclorometano (3:1:3 $\mathrm{v} / \mathrm{v}$ ), P.f. $=79,7-80,4{ }^{\circ} \mathrm{C}, \mathrm{R}_{\mathrm{f}}$ 0,29 (hexano-acetato de etila-diclorometano 3:1:3 v/v). IV (ATR) $v_{\max } / \mathrm{cm}^{-1}: 3111,3062,2949,2911$, 1590, 1518, 1465, 1348, 1227, 1139, 1014, 907, 849, 798, 713, 592. RMN ${ }^{1} \mathrm{H}\left(300 \mathrm{MHz}, \mathrm{CDCl}_{3}\right.$ ) $\delta: 2,19$ (quint, $2 \mathrm{H}, J=6,9 \mathrm{~Hz}$ ); 2,94 $(\mathrm{t}, 2 \mathrm{H}, J=7,5 \mathrm{~Hz}) ; 3,31(\mathrm{~d}, 2 \mathrm{H}, J=6,7 \mathrm{~Hz}) ; 3,81(\mathrm{~s}, 3 \mathrm{H}) ; 4,01(\mathrm{t}$, $2 \mathrm{H}, J=6,3 \mathrm{~Hz}) ; 5,00-5,12(\mathrm{~m}, 2 \mathrm{H}) ; 5,59$ (s, 2H); 5,94 (ddt $\mathrm{ap}_{1}, 1 \mathrm{H}$, $J_{1}=16,8 \mathrm{~Hz}, J_{2}=10,2 \mathrm{~Hz}$ e $\left.J_{3}=6,7 \mathrm{~Hz}\right) ; 6,65-6,73(\mathrm{~m}, 2 \mathrm{H}) ; 6,77$ (d, $1 \mathrm{H}, J=7,8 \mathrm{~Hz}) ; 7,31-7,38(\mathrm{~m}, 3 \mathrm{H}) ; 8,19(\mathrm{~d}, 2 \mathrm{H}, J=8,7 \mathrm{~Hz})$. $\mathrm{RMN}{ }^{13} \mathrm{C}\left(75 \mathrm{MHz}, \mathrm{CDCl}_{3}\right) \delta: 22,1 ; 28,6 ; 39,7 ; 52,9 ; 55,8 ; 68,0$; 112,$4 ; 113,4 ; 115,6 ; 120,4 ; 121,3 ; 124,2 ; 128,4 ; 133,0 ; 137,5$; 141,$9 ; 146,6 ; 147,9 ; 148,3 ; 149,3$. HRMS $\left(\mathrm{M}+\mathrm{H}^{+}\right)$: calculado para $\mathrm{C}_{22} \mathrm{H}_{25} \mathrm{~N}_{4} \mathrm{O}_{4}$ : 409,18758; encontrado: 409,18677. HRMS $\left(\mathrm{M}+\mathrm{Na}^{+}\right)$: calculado para $\mathrm{C}_{22} \mathrm{H}_{24} \mathrm{~N}_{4} \mathrm{NaO}_{4}$ : 431,16952; encontrado: 431,16867.

Dados para 4-(3-(4-alil-2-metoxifenoxi)propil)-1-(4-metoxibenzil)-1 $H$-1,2,3-triazol (7g): Sólido branco, purificado por cromatografia em coluna eluída com hexano-acetato de etila-diclorometano (3:1:3 v/v), P.f. $=88,9-90,4{ }^{\circ} \mathrm{C}, \mathrm{R}_{\mathrm{f}}=0,22$ (hexano-acetato de etila-diclorometano $3: 1: 3 \mathrm{v} / \mathrm{v}$ ). IV (ATR) $v_{\max } / \mathrm{cm}^{-1}: 3114,3062,3005,2951,2868$, 1587, 1512, 1455, 1295, 1251, 1227, 1131, 1032, 1015, 917, 801, $749,652 . \mathrm{RMN}^{1} \mathrm{H}\left(300 \mathrm{MHz}, \mathrm{CDCl}_{3}\right) \delta: 2,16$ (quint, $2 \mathrm{H}, J=7,0 \mathrm{~Hz}$ ), $2,88(\mathrm{t}, 2 \mathrm{H}, J=7,5 \mathrm{~Hz}), 3,32(\mathrm{~d}, 2 \mathrm{H}, J=6,6 \mathrm{~Hz}), 3,80(\mathrm{~s}, 3 \mathrm{H}), 3,82$ (s, 3H), 4,01 (t, 2H, J =6,4 Hz), 5,00-5,11 (m, 2H), 5,40 (s, 2H), $5,95\left(\mathrm{ddt}_{\mathrm{ap}}, 1 \mathrm{H}, J_{1}=16,8 \mathrm{~Hz}, J_{2}=9,9 \mathrm{~Hz}\right.$ e $\left.J_{3}=6,6 \mathrm{~Hz}\right), 6,65-6,73(\mathrm{~m}$,
2H), 6,77 (d, 1H, $J=7,9 \mathrm{~Hz}), 6,87(\mathrm{~d}, 2 \mathrm{H}, J=8,6 \mathrm{~Hz}), 7,15-7,23$ (m, $3 \mathrm{H}) . \mathrm{RMN}^{13} \mathrm{C}\left(75 \mathrm{MHz}, \mathrm{CDCl}_{3}\right) \delta: 22,1,28,7,39,7,53,5,55,3,55,8$, $68,1,112,3,113,4,114,4,115,5,120,4,120,6,126,8,129,5,132,9$, $137,6,146,6,147,6,149,3,159,8$. HRMS $\left(\mathrm{M}+\mathrm{H}^{+}\right)$: calculado para $\mathrm{C}_{23} \mathrm{H}_{28} \mathrm{~N}_{3} \mathrm{O}_{3}: 394,21307$; encontrado: 394,21225. HRMS (M+Na+): calculado para $\mathrm{C}_{23} \mathrm{H}_{27} \mathrm{~N}_{3} \mathrm{NaO}_{3}$ : 416,19501; encontrado: 416,19415.

Dados para 4-(3-(4-alil-2-metoxifenoxi)propil)-1-(4-(trifluorometoxi)benzil)-1 $H$-1,2,3-triazol (7h): Sólido branco, purificado por cromatografia em coluna eluída com hexano-acetato de etila-diclorometano $(3: 1: 3 \mathrm{v} / \mathrm{v})$, P.f. $=93,4-94,9^{\circ} \mathrm{C}, \mathrm{R}_{\mathrm{f}}=0,49$ (hexano-acetato de etila-diclorometano 3:1:3 v/v). IV (ATR) $v_{\max } / \mathrm{cm}^{-1}: 3118,3063,2949$, 2878, 1590, 1511, 1454, 1259, 1225, 1138, 1034, 1014, 911, 800, $743,652 . \mathrm{RMN}^{1} \mathrm{H}\left(300 \mathrm{MHz}, \mathrm{CDCl}_{3}\right) \delta: 2,18$ (quint, $2 \mathrm{H}, J=6,9 \mathrm{~Hz}$ ); $2,91(\mathrm{t}, 2 \mathrm{H}, J=7,5 \mathrm{~Hz}) ; 3,32(\mathrm{~d}, 2 \mathrm{H}, J=6,7 \mathrm{~Hz}) ; 3,81(\mathrm{~s}, 3 \mathrm{H}) ; 4,01$ (t, 2H, $J=6,3 \mathrm{~Hz}) ; 5,01-5,13(\mathrm{~m}, 2 \mathrm{H}) ; 5,48(\mathrm{~s}, 2 \mathrm{H}) ; 5,95\left(\mathrm{ddt}_{\mathrm{ap}}, 1 \mathrm{H}\right.$, $J_{1}=16,8 \mathrm{~Hz}, J_{2}=10,2 \mathrm{~Hz}$ e $\left.J_{3}=6,7 \mathrm{~Hz}\right) ; 6,65-6,73(\mathrm{~m}, 2 \mathrm{H}) ; 6,77(\mathrm{~d}$, $1 \mathrm{H}, J=7,9 \mathrm{~Hz}) ; 7,19(\mathrm{~d}, 2 \mathrm{H}, J=8,6 \mathrm{~Hz}) ; 7,23-7,31(\mathrm{~m}, 3 \mathrm{H}) . \mathrm{RMN}^{13} \mathrm{C}$ $\left(75 \mathrm{MHz}, \mathrm{CDCl}_{3}\right) \delta: 22,1 ; 28,7 ; 39,7 ; 53,0 ; 55,8 ; 68,0 ; 112,3 ; 113,4$; 115,$6 ; 120,3(\mathrm{q}, J=257,0 \mathrm{~Hz}) ; 120,4 ; 121,0 ; 121,4 ; 129,3 ; 133,0$; 133,$6 ; 137,6 ; 146,6 ; 148,0 ; 149,2-149,3(\mathrm{~m}) ; 149,4 \mathrm{HRMS}\left(\mathrm{M}+\mathrm{H}^{+}\right)$: calculado para $\mathrm{C}_{23} \mathrm{H}_{25} \mathrm{~F}_{3} \mathrm{~N}_{3} \mathrm{O}_{3}$ : 448,18480; encontrado: 448,18399. HRMS $\left(\mathrm{M}+\mathrm{Na}^{+}\right)$: calculado para $\mathrm{C}_{23} \mathrm{H}_{24} \mathrm{~F}_{3} \mathrm{~N}_{3} \mathrm{NaO}_{3}$ : 470,16675; encontrado: 470,16602 .

Dados para 4-(3-(4-alil-2-metoxifenoxi)propil)-1-(4-(trifluorometil)benzil)-1H-1,2,3-triazol (7i): Sólido branco, purificado por cromatografia em coluna eluída com hexano-acetato de etila-diclorometano $(3: 1: 3 \mathrm{v} / \mathrm{v})$, P.f. $=111,3-112,5^{\circ} \mathrm{C}, \mathrm{R}_{\mathrm{f}}=0,50$ (hexano-acetato de etila-diclorometano $3: 1: 3 \mathrm{v} / \mathrm{v}$ ). IV (ATR) $v_{\max } / \mathrm{cm}^{-1}: 3110,3062,2947$, 2910, 1589, 1516, 1466, 1421, 1328, 1263, 1224, 1142, 1116, 1014, $908,798,740,669 . \mathrm{RMN}^{1} \mathrm{H}\left(300 \mathrm{MHz}, \mathrm{CDCl}_{3}\right) \delta: 2,19$ (quint, $2 \mathrm{H}$, $J=6,9 \mathrm{~Hz}) ; 2,92(\mathrm{t}, 2 \mathrm{H}, J=7,5 \mathrm{~Hz}) ; 3,32(\mathrm{~d}, 2 \mathrm{H}, J=6,7 \mathrm{~Hz}) ; 3,81$ $(\mathrm{s}, 3 \mathrm{H}) ; 4,02(\mathrm{t}, 2 \mathrm{H}, J=6,3 \mathrm{~Hz}) ; 5,00-5,13(\mathrm{~m}, 2 \mathrm{H}) ; 5,54(\mathrm{~s}, 2 \mathrm{H}) ; 5,94$ $\left(\mathrm{ddt}_{\mathrm{ap}}, 1 \mathrm{H}, J_{1}=16,8 \mathrm{~Hz}, J_{2}=10,2 \mathrm{~Hz}\right.$ e $\left.J_{3}=6,74 \mathrm{~Hz}\right) ; 6,65-6,73(\mathrm{~m}$, $2 \mathrm{H}) ; 6,77(\mathrm{~d}, 1 \mathrm{H}, J=7,9 \mathrm{~Hz}) ; 7,28(\mathrm{~s}, 1 \mathrm{H}) ; 7,32(\mathrm{~d}, 2 \mathrm{H}, J=8,1 \mathrm{~Hz})$; $7,61$ (d, $2 \mathrm{H}, J=8,1 \mathrm{~Hz})$. RMN ${ }^{13} \mathrm{C}\left(75 \mathrm{MHz}, \mathrm{CDCl}_{3}\right) \delta: 22,1 ; 28,8$; 39,$7 ; 53,2 ; 55,9 ; 68,0 ; 112,3 ; 113,4 ; 115,6 ; 120,4 ; 121,1 ; 123,8$ (q, $J=270,8 \mathrm{~Hz}) ; 126,0(\mathrm{q}, J=3,8 \mathrm{~Hz}) ; 128,0 ; 130,9$ (q, $J=32,5 \mathrm{~Hz})$; 133,$0 ; 137,6 ; 138,8 ; 146,7 ; 148,1 ; 149,4$. HRMS $\left(\mathrm{M}+\mathrm{H}^{+}\right)$: calculado para $\mathrm{C}_{23} \mathrm{H}_{25} \mathrm{~F}_{3} \mathrm{~N}_{3} \mathrm{O}_{2}$ : 432,18989; encontrado: 432,18903. HRMS $\left(\mathrm{M}+\mathrm{Na}^{+}\right)$: calculado para $\mathrm{C}_{23} \mathrm{H}_{24} \mathrm{~F}_{3} \mathrm{~N}_{3} \mathrm{NaO}_{2}$ : 454,17183; encontrado: 454,17093 .

Dados para 4-(3-(4-alil-2-metoxifenoxi)propil)-1-(3,4-difluorobenzil)-1H-1,2,3-triazol (7j): Sólido branco, purificado por cromatografia em coluna eluída com hexano-acetato de etila-diclorometano $(3: 1: 3 \mathrm{v} / \mathrm{v})$, P.f. $=97,1-97,8^{\circ} \mathrm{C}, \mathrm{R}_{\mathrm{f}}=0,41$ (hexano-acetato de etila-diclorometano 3:1:3 v/v). IV (ATR) $v_{\max } / \mathrm{cm}^{-1}: 3111,3061,2949$, $1588,1515,1454,1261,1288,1228,1141,1017,915,754 . \mathrm{RMN}^{1} \mathrm{H}$ (300 MHz, $\left.\mathrm{CDCl}_{3}\right) \mathrm{RMN}^{1} \mathrm{H}\left(300 \mathrm{MHz}, \mathrm{CDCl}_{3}\right) \delta: 2,18$ (quint, $2 \mathrm{H}$, $J=6,9 \mathrm{~Hz}) ; 2,92$ (t, 2H, $J=7,5 \mathrm{~Hz}) ; 3,32$ (d, 2H, $J=6,7 \mathrm{~Hz}) ; 3,82$ (s, 3H); 4,01 (t, 2H, $J=6,3 \mathrm{~Hz}) ; 5,00-5,12(\mathrm{~m}, 2 \mathrm{H}) ; 5,43(\mathrm{~s}, 2 \mathrm{H})$; $5,95\left(\mathrm{ddt}_{\mathrm{ap}}, 1 \mathrm{H}, J_{1}=16,8 \mathrm{~Hz}, J_{2}=10,2 \mathrm{~Hz}\right.$ e $\left.J_{3}=6,7 \mathrm{~Hz}\right) ; 6,65-6,71$ $(\mathrm{m}, 2 \mathrm{H}) ; 6,77$ (d, 1H, $J=7,9 \mathrm{~Hz}) ; 6,92-7,20(\mathrm{~m}, 3 \mathrm{H}) ; 7,27$ (s, 1H). $\mathrm{RMN}{ }^{13} \mathrm{C}\left(75 \mathrm{MHz}, \mathrm{CDCl}_{3}\right) \delta: 22,1 ; 28,7 ; 39,7 ; 52,5 ; 55,9(\mathrm{dl}$, $J=8,3 \mathrm{~Hz}) ; 68,0 ; 112,5 ; 113,4 ; 115,6 ; 117,0$ (d, $J=17,3 \mathrm{~Hz}) ; 117,9$ $(\mathrm{d}, J=17,3 \mathrm{~Hz}) ; 120,4 ; 120,9 ; 124,0\left(\mathrm{dd}, J_{1}=6,8 \mathrm{~Hz}\right.$ e $\left.J_{2}=3,8 \mathrm{~Hz}\right)$; $131,8-131,9$ (m); 133,0;137,5; 146,5; 148,1; 149,3; [148,6-148,9 (m), 151,9-152,2 (m)]. HRMS $\left(\mathrm{M}+\mathrm{H}^{+}\right)$: calculado para $\mathrm{C}_{22} \mathrm{H}_{24} \mathrm{~F}_{2} \mathrm{~N}_{3} \mathrm{O}_{2}$ : 400,18366; encontrado: 400,18274. HRMS $\left(\mathrm{M}+\mathrm{Na}^{+}\right)$: calculado para $\mathrm{C}_{22} \mathrm{H}_{23} \mathrm{~F}_{2} \mathrm{~N}_{3} \mathrm{NaO}_{2}$ : 422,16560; encontrado: 422,16473.

Dados para 4-(3-(4-alil-2-metoxifenoxi)propil)-1-(4-metilbenzil)-1H-1,2,3-triazol (7k): Sólido branco, purificado por cromatografia em coluna eluída com hexano-acetato de etila-diclorometano $(3: 1: 3 \mathrm{v} / \mathrm{v})$, P.f. $=59,6-60,9{ }^{\circ} \mathrm{C}, \mathrm{R}_{\mathrm{f}}=0,42$ (hexano-acetato de 
etila-diclorometano 3:1:3 v/v). IV (ATR) $v_{\max } / \mathrm{cm}^{-1}: 3111,3061$, 2953, 2916, 2880, 1588, 1514, 1449, 1420, 1260, 1228, 1140, 1014, $904,845,799,75,720,650 . \mathrm{RMN}{ }^{1} \mathrm{H}\left(300 \mathrm{MHz}, \mathrm{CDCl}_{3}\right) \delta: 2,16$ (quint, $2 \mathrm{H}, J=7,0 \mathrm{~Hz}) ; 2,34(\mathrm{~s}, 3 \mathrm{H}) ; 2,88(\mathrm{t}, 2 \mathrm{H}, J=7,5 \mathrm{~Hz}) ; 3,32$ $(\mathrm{d}, 2 \mathrm{H}, J=6,7 \mathrm{~Hz}) ; 3,81(\mathrm{~s}, 3 \mathrm{H}) ; 4,01$ (t, 2H, $J=6,4 \mathrm{~Hz}) ; 5,00-5,13$ $(\mathrm{m}, 2 \mathrm{H}) ; 5,43(\mathrm{~s}, 2 \mathrm{H}) ; 5,95\left(\mathrm{ddt}_{\mathrm{ap}}, 1 \mathrm{H}, J_{l}=16,8 \mathrm{~Hz}, J_{2}=10,2 \mathrm{~Hz}\right.$ e $\left.J_{3}=6,7 \mathrm{~Hz}\right) ; 6,65-6,73(\mathrm{~m}, 2 \mathrm{H}) ; 6,77(\mathrm{~d}, 1 \mathrm{H}, J=7,9 \mathrm{~Hz}) ; 7,10$ $7,18(\mathrm{~m}, 4 \mathrm{H}) ; 7,21(\mathrm{~s}, 1 \mathrm{H}) . \mathrm{RMN}{ }^{13} \mathrm{C}\left(75 \mathrm{MHz}, \mathrm{CDCl}_{3}\right) \delta: 21,0$; 22,$1 ; 28,7 ; 39,7 ; 53,7 ; 55,8 ; 68,0112,3 ; 113,4 ; 115,5 ; 120,4 ; 120,7$; 127,$9 ; 129,6 ; 131,7 ; 132,8 ; 137,5 ; 138,4 ; 146,6 ; 147,6 ; 149,3$. HRMS $\left(\mathrm{M}+\mathrm{H}^{+}\right)$: calculado para $\mathrm{C}_{23} \mathrm{H}_{28} \mathrm{~N}_{3} \mathrm{O}_{2}: 378,21815$; encontrado: 378,21719 . HRMS $\left(\mathrm{M}+\mathrm{Na}^{+}\right)$: calculado para $\mathrm{C}_{23} \mathrm{H}_{27} \mathrm{~N}_{3} \mathrm{NaO}_{2}$ : 400,20010; encontrado: 400,19922.

Dados para 4-(3-(4-alil-2-metoxifenoxi)propil)-1-(2-bromobenzil)-1H-1,2,3-triazol (7l): Sólido branco, purificado por cromatografia em coluna eluída com hexano-acetato de etila-diclorometano (3:1:3 v/v), P.f. $=47,8-49,1^{\circ} \mathrm{C}, \mathrm{R}_{\mathrm{f}}=0,61$ (hexano-acetato de etila-diclorometano 3:1:3 v/v). IV (ATR) $v_{\max } / \mathrm{cm}^{-1}: 3104,3054,2931$, 1587, 1511, 1467, 1419, 1230, 1135, 1027, 948, 804, 744, 653. $\mathrm{RMN}{ }^{1} \mathrm{H}\left(300 \mathrm{MHz}, \mathrm{CDCl}_{3}\right) \delta: 2,19$ (quint, $\left.2 \mathrm{H}, J=7,0 \mathrm{~Hz}\right) ; 2,92$ $(\mathrm{t}, 2 \mathrm{H}, J=7,5 \mathrm{~Hz}) ; 3,32(\mathrm{~d}, 2 \mathrm{H}, J=6,7 \mathrm{~Hz}) ; 3,82(\mathrm{~s}, 3 \mathrm{H}) ; 4,02(\mathrm{t}$, $2 \mathrm{H}, J=6,4 \mathrm{~Hz}) ; 5,00-5,13(\mathrm{~m}, 2 \mathrm{H}) ; 5,61(\mathrm{~s}, 2 \mathrm{H}) ; 5,95\left(\mathrm{ddt}_{\mathrm{ap}}, 1 \mathrm{H}\right.$, $J_{l}=16,8 \mathrm{~Hz}, J_{2}=10,2 \mathrm{~Hz}$ e $\left.J_{3}=6,7 \mathrm{~Hz}\right) ; 6,65-6,73(\mathrm{~m}, 2 \mathrm{H}) ; 6,78$ $(\mathrm{d}, 1 \mathrm{H}, J=7,8 \mathrm{~Hz}) ; 7,08\left(\mathrm{dd}, 1 \mathrm{H}, J_{1}=7,5 \mathrm{~Hz}\right.$ e $\left.J_{2}=1.6 \mathrm{~Hz}\right) ; 7,15$ 7,31 (m, 2H); 7,35 (s, 1H); 7,59 (dd, 1H, $J_{1}=7,8 \mathrm{~Hz}$ e $\left.J_{2}=1,1 \mathrm{~Hz}\right)$. $\mathrm{RMN}{ }^{13} \mathrm{C}\left(75 \mathrm{MHz}, \mathrm{CDCl}_{3}\right) \delta: 22,1 ; 28,7 ; 39,7 ; 53,6 ; 55,8 ; 68,1$; 112,$3 ; 113,4 ; 115,5 ; 120,3 ; 121,3 ; 123,2 ; 128,1 ; 130,1 ; 130,2$; 132,$9 ; 133,1 ; 134,4 ; 137,6 ; 146,6 ; 147,7 ; 149,3$. HRMS $\left(\mathrm{M}+\mathrm{H}^{+}\right)$: calculado para $\mathrm{C}_{22} \mathrm{H}_{25} \mathrm{BrN}_{3} \mathrm{O}_{2}$ : 442,11301; encontrado: 442,11197. HRMS $\left(\mathrm{M}+\mathrm{Na}^{+}\right)$: calculado para $\mathrm{C}_{22} \mathrm{H}_{24} \mathrm{BrN}_{3} \mathrm{NaO}_{2}$ : 464,09496; encontrado: 464,09390.

Dados para 4-(3-(4-alil-2-metoxifenoxi)propil)-1-(3-bromobenzil)-1H-1,2,3-triazol (7m): Sólido branco, purificado por cromatografia em coluna eluída com hexano-acetato de etila-diclorometano $(3: 1: 3 \mathrm{v} / \mathrm{v})$, P.f. $=65,9-67,1^{\circ} \mathrm{C}, \mathrm{R}_{\mathrm{f}}=0,32$ (hexano-acetato de etila-diclorometano 3:1:3 v/v). IV (ATR) $v_{\max } / \mathrm{cm}^{-1}: 3110,3062,2949$, 2881, 1589, 1514, 1462, 1260, 1227, 1140, 1013, 798, 726, 689, 586. $\mathrm{RMN}{ }^{1} \mathrm{H}\left(300 \mathrm{MHz}, \mathrm{CDCl}_{3}\right) \delta: 2,18$ (quint, $2 \mathrm{H}, J=6,9 \mathrm{~Hz}$ ); $2,91(\mathrm{t}, 2 \mathrm{H}, J=7,5 \mathrm{~Hz}) ; 3,32(\mathrm{~d}, 2 \mathrm{H}, J=6,7 \mathrm{~Hz}) ; 3,82(\mathrm{~s}, 3 \mathrm{H}) ; 4,02$ $(\mathrm{t}, 2 \mathrm{H}, J=6,3 \mathrm{~Hz}) ; 5,00-5,13(\mathrm{~m}, 2 \mathrm{H}) ; 5,44(\mathrm{~s}, 2 \mathrm{H}) ; 5,94\left(\mathrm{ddt}_{\mathrm{ap}}, 1 \mathrm{H}\right.$, $J_{l}=16,8 \mathrm{~Hz}, J_{2}=10,2 \mathrm{~Hz}$ e $\left.J_{3}=6,7 \mathrm{~Hz}\right) ; 6,65-6,73(\mathrm{~m}, 2 \mathrm{H}) ; 6,78$ (d, 1H, $J=7,9 \mathrm{~Hz})$; [7,10-7,30 (m), $7.38(\mathrm{sl}), 7,46\left(\mathrm{~d}_{\mathrm{ap}}, J=7,8 \mathrm{~Hz}\right.$, 5H]. RMN ${ }^{13} \mathrm{C}\left(75 \mathrm{MHz}, \mathrm{CDCl}_{3}\right) \delta: 22,1 ; 28,7 ; 39,7 ; 53,1 ; 55,8$; 68,$0 ; 112,3 ; 113,4 ; 115,6 ; 120,4 ; 121,0 ; 123,0 ; 126,4 ; 130,6 ; 130,8$; 131,$7 ; 132,9 ; 137,1 ; 137,6 ; 146,6 ; 148,0 ; 149,3$. HRMS $\left(\mathrm{M}+\mathrm{H}^{+}\right)$: calculado para for $\mathrm{C}_{22} \mathrm{H}_{25} \mathrm{BrN}_{3} \mathrm{O}_{2}$ : 442,11301; encontrado: 442,11218. HRMS $\left(\mathrm{M}+\mathrm{Na}^{+}\right)$: calculado para for $\mathrm{C}_{22} \mathrm{H}_{24} \mathrm{BrN}_{3} \mathrm{NaO}_{2}$ : 464,09496; encontrado: 464,09412.

\section{Ensaios biológicos}

\section{Cultivo de linhagens celulares}

As linhagens celulares HL60 (leucemia mieloide aguda), Nalm6 (leucemia linfoide aguda) e B16F10 (melanoma metastático murino) foram cultivadas em meio RPMI-1640 (Roswell Park Memorial Institute Medium) $\mathrm{pH}$ 7,4 suplementado com $10 \% \mathrm{v} / \mathrm{v}$ de soro fetal bovino, $100 \mathrm{U} / \mathrm{mL}$ de penicilina e $100 \mu \mathrm{g} / \mathrm{mL}$ de estreptomicina. As células cresceram em garrafas de cultivo celular sob atmosfera umidificada com $5 \%$ de $\mathrm{CO}_{2}$.

\section{Ensaio de Viabilidade Celular (MTT)}

Células HL60 e Nalm6 (7,0 x 10 células/poço) e B16F10 (1,0 x $10^{4}$ células/poço) foram semeadas em placas de 96 poços, cada um contendo $100 \mu \mathrm{L}$ de meio RPMI completo e $100 \mu \mathrm{L}$ de solução de cada composto em diferentes concentrações. Os compostos foram diluídos em meio RPMI contendo $10 \% \mathrm{v} / \mathrm{v}$ de soro fetal bovino e $0,4 \% \mathrm{v} / \mathrm{v}$ de DMSO (Sigma). Células tratadas com $0,4 \%$ v/v de DMSO (veículo) foram utilizadas como controle. Após 48 horas de incubação, $10 \mu \mathrm{L}$ de MTT ( $5 \mathrm{mg} / \mathrm{mL}$, Sigma) foram adicionados em cada poço. Após 3 horas a $37^{\circ} \mathrm{C}$, a solução contendo MTT foi removida dos poços e então foram adicionados $100 \mu \mathrm{L} /$ poço de DMSO para solubilização do formazan. Finalmente, a absorbância foi avaliada em leitora de microplacas (Spectra Max M5, Molecular Devices).

\section{RESULTADOS E DISCUSSÃO}

\section{Síntese dos compostos triazólicos derivados do eugenol}

A primeira etapa envolvida na preparação dos compostos derivados do eugenol contendo núcleos 1,2,3-triazólicos correspondeu à extração do eugenol (1). Este composto foi obtido por hidrodestilação do cravo da índia e purificado por cromatografia em coluna de sílica gel. Em seguida, o eugenol (1) foi submetido a reações de alquilação resultando na obtenção dos derivados (2) e (4) conforme apresentado no Esquema 1. ${ }^{16}$

Para a síntese dos compostos triazólicos derivados do eugenol foi necessária a preparação de várias azidas benzílicas (estrutura geral (5) no Esquema 2) que foram obtidas em duas etapas a partir dos álcoois benzílicos correspondentes, de acordo com a metodologia descrita na literatura. ${ }^{17}$

A etapa chave envolvida na síntese dos compostos triazólicos derivados do eugenol correspondeu à reação de cicloadição entre um alcino e uma azida catalisada por cobre (I) (CuAAC), também conhecida como reação "click". ${ }^{18-23}$ Assim, os compostos 6a-6n e 7a-7m foram preparados a partir dos compostos alquilados (2 e 4) e as azidas benzílicas (5), e obtidos com rendimentos sinteticamente úteis $(77 \%-91 \%)$ (Esquema 3$)$.

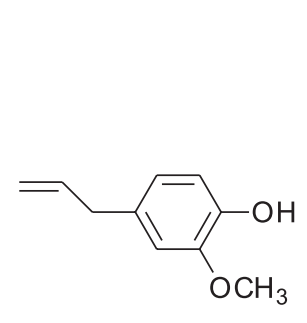

(1)

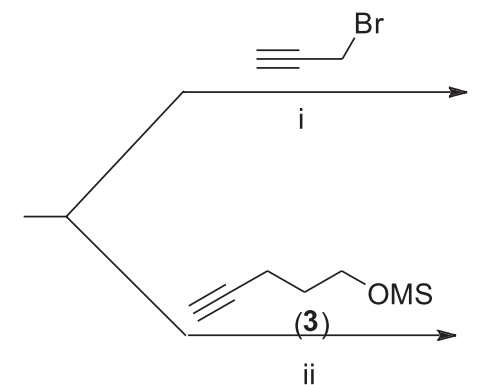

ii

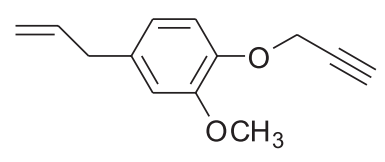

(2); $87 \%$

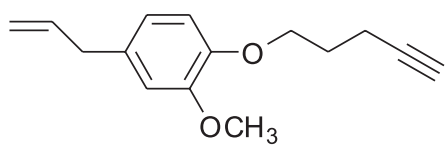

$(4) ; 78 \%$

Esquema 1. i) $\mathrm{NaOH}, \mathrm{CH}_{3} \mathrm{OH}, 40^{\circ} \mathrm{C}$; acetonitrila, t.a; ii) $\mathrm{NaOH}, \mathrm{CH}_{3} \mathrm{OH}, 40^{\circ} \mathrm{C}$; acetonitrila, $70^{\circ} \mathrm{C}$ 


$$
\mathrm{Ar}-\mathrm{CH}_{2} \mathrm{OH} \underset{\mathrm{iii}}{\longrightarrow} \mathrm{Ar}-\mathrm{CH}_{2} \mathrm{OSO}_{2} \mathrm{CH}_{3} \longrightarrow \mathrm{iv} \longrightarrow \mathrm{CH}_{2} \mathrm{~N}_{3}
$$

$\mathrm{Ar}=$ fenila; 4-fluorofenila; 4-clorofenila; 4-bromofenila; 4-iodofenila; 4-nitrofenila; 4-metoxif enila; 4trifluorometoxif enila; 4-trifluorometilfenila; 3,4-difluorofenila; 2,5-diclorofenila; 4-metilfenila; 2bromofenila; 3-bromofenila.

Esquema 2. iii) $\mathrm{Et}_{3} \mathrm{~N}, \mathrm{CH}_{3} \mathrm{SO}_{2} \mathrm{Cl}, \mathrm{CH}_{2} \mathrm{Cl}_{2},-50^{\circ} \mathrm{C}$; iv) $\mathrm{NaN}_{3}, \mathrm{DMSO}$, t.a.

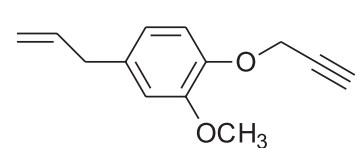

(2)

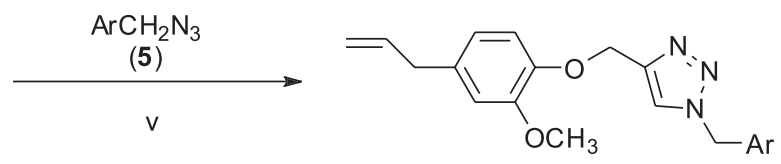

$(6 a-6 n)$

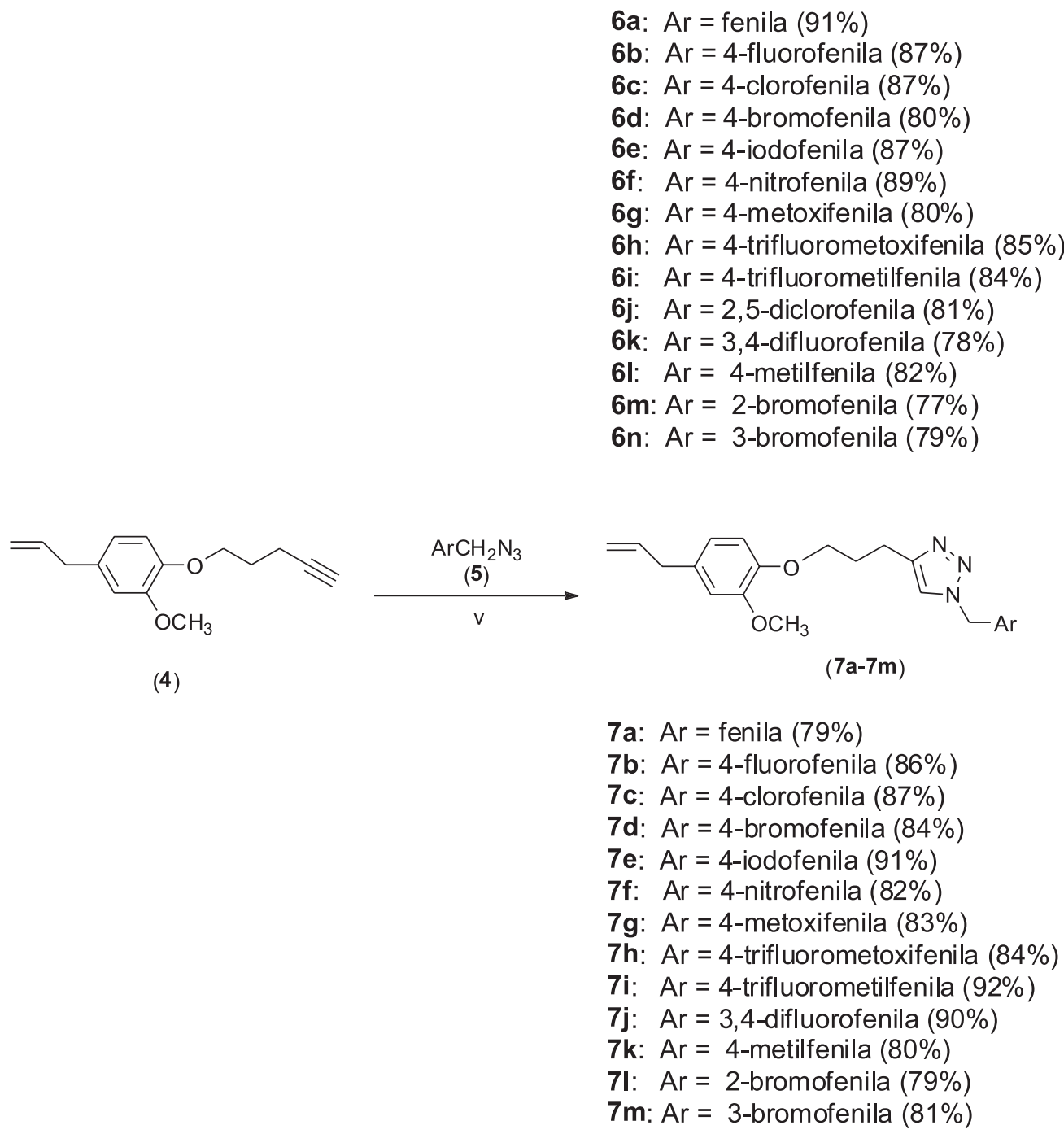

Esquema 3. v) ascorbato de sódio (40 mol\%), $\mathrm{CuSO}_{4} \cdot 5 \mathrm{H}_{2} \mathrm{O}(20 \mathrm{~mol} \%)$, $\mathrm{EtOH} / \mathrm{H}_{2} \mathrm{O}(1: 1 \mathrm{v} / \mathrm{v})$

Os compostos triazólicos foram obtidos empregando-se condições de reação similares àquelas previamente descritas na literatura. ${ }^{17} \mathrm{As}$ reações, de modo geral, duraram menos do que um minuto e não foram observadas variações de velocidade das transformações utilizando-se azidas benzílicas contendo grupos doadores ou retiradores de elétrons ligados ao anel aromático. O alquino terminal utilizado (2 ou 4) também não influenciou a velocidade dos processos "click".

Todos os compostos triazólicos foram completamente caracterizados pelas espectroscopias de $\mathrm{RMN}\left({ }^{1} \mathrm{H} \mathrm{e}{ }^{13} \mathrm{C}\right)$ e no infravermelho
(IV) bem como pela espectrometria de massas. Nos espectros de RMN de ${ }^{1} \mathrm{H}$, o sinal atribuído ao hidrogênio presente no anel triazólico foi observado como um simpleto dentro da faixa de 7,20-7,67 ppm. Os sinais referentes aos átomos de hidrogênios metilênicos ligados ao oxigênio e ao nitrogênio também foram observados como simpletos nos espectros de RMN de ${ }^{1} \mathrm{H}$. No espectro de $\mathrm{RMN}$ de ${ }^{13} \mathrm{C}$ foi observado que o número de sinais presentes e os deslocamentos químicos são compatíveis com as estruturas das substâncias. Nos espectros no IV, as bandas esperadas para os grupos funcionais foram 
observadas. As fórmulas moleculares dos derivados triazólicos do eugenol 6a-6n e 7a-7m foram confirmadas pela espectrometria de massas de alta resolução. Cabe destacar que a síntese dos compostos foi planejada de modo a avaliar o efeito dos diferentes grupos benzila ligados à porção triazólica (Esquema 3 ) bem como do tamanho da cadeia alquílica (ligando a porção eugenol à porção triazólica) sobre a atividade citotóxica dos compostos.

\section{Avaliação da atividade citotóxica dos compostos triazólicos derivados do eugenol}

Os compostos triazólicos $\mathbf{6 a - 6 n}$ e $\mathbf{7 a - 7} \mathbf{m}$ foram inicialmente avaliados com respeito aos seus efeitos citotóxicos contra a linhagem leucêmica HL60 (leucemia mieloide aguda). Esta linhagem foi tratada com diferentes concentrações dos compostos $\left(100,50\right.$ e $\left.25 \mu \mathrm{mol} \mathrm{L}^{-1}\right)$ por 48 horas. Os testes foram realizados em triplicata e os resultados obtidos para a viabilidade celular estão apresentados na Tabela 1.
Não foi possível estabelecer uma correlação entre estruturas químicas e atividade biológica. Os resultados apresentados mostram que os compostos $\mathbf{6 n}, \mathbf{7 a}, \mathbf{7 c}, \mathbf{7 e}$ e $\mathbf{7} \mathbf{m}$ foram os mais ativos e apresentaram uma redução igual ou superior a $80 \%$ de células viáveis nos tratamentos a $100 \mu \mathrm{mol} \mathrm{L} \mathrm{L}^{-1}$.

Devido ao efeito citotóxico mais pronunciado para estas substâncias, elas foram consideradas promissoras e tiveram suas atividades citotóxicas avaliadas contra as linhagens celulares HL60, Nalm6 e $\mathrm{B} 16 \mathrm{~F} 10$ para determinação de valores de $\mathrm{IC}_{50}$. Neste caso, as linhagens foram tratadas com diferentes concentrações dos compostos $(200,150$, $125,100,75,50$ e $25 \mu \mathrm{mol} \mathrm{L}-1)$, por 48 horas. Esses testes foram realizados em triplicata e os resultados obtidos são apresentados na Tabela 2.

Comparando-se os valores de $\mathrm{IC}_{50}$ apresentados na Tabela 2, observa-se, como tendência geral, que a linhagem mieloide (HL60) apresentou maior sensibilidade ao efeito citotóxico dos compostos em relação à linhagem linfoide (Nalm6) e murina (B16F10). Além disso, observa-se que dos cinco compostos mais ativos, quatro (7a, 7c, 7e,

Tabela 1. Efeito dos compostos triazólicos derivados do eugenol sobre a viabilidade (\%) da linhagem celular HL60, após 48 horas de exposição aos compostos (média \pm desvio padrão)

\begin{tabular}{|c|c|c|c|c|}
\hline \multirow{2}{*}{ Composto* } & \multirow{2}{*}{$\mathrm{Ar}$} & \multicolumn{3}{|c|}{ Viabilidade $(\%)^{* *}$} \\
\hline & & $25 \mu \mathrm{mol} \mathrm{L}^{-1}$ & $50 \mu \mathrm{mol} \mathrm{L}{ }^{-1}$ & $100 \mu \mathrm{mol} \mathrm{L}^{-1}$ \\
\hline $6 a$ & Fenila & $75,92 \pm 16,21$ & $77,73 \pm 12,22$ & $69,65 \pm 9,62$ \\
\hline $6 \mathbf{b}$ & 4-fluorofenila & $67,98 \pm 13,09$ & $64,12 \pm 3,08$ & $54,89 \pm 11,22$ \\
\hline $6 c$ & 4-clorofenila & $75,25 \pm 3,53$ & $76,87 \pm 11,19$ & $83,68 \pm 6,07$ \\
\hline 6d & 4-bromofenila & $75,72 \pm 1,83$ & $81,12 \pm 2,81$ & $82,68 \pm 9,84$ \\
\hline $6 e$ & 4-iodofenila & $78,23 \pm 6,39$ & $81,57 \pm 12,93$ & $86,54 \pm 18,31$ \\
\hline $6 f$ & 4-nitrofenila & $74,31 \pm 3,11$ & $72,03 \pm 1,53$ & $78,63 \pm 0,40$ \\
\hline $6 \mathrm{~g}$ & 4-metoxifenila & $71,78 \pm 9,36$ & $74,76 \pm 12,12$ & $78,80 \pm 3,64$ \\
\hline $6 \mathrm{~h}$ & 4-trifluorometoxifenila & $86,84 \pm 16,65$ & $76,06 \pm 6,68$ & $73,70 \pm 16,80$ \\
\hline $6 \mathbf{i}$ & 4-triflorometila & $79,77 \pm 1,55$ & $80,68 \pm 7,02$ & $77,10 \pm 6,15$ \\
\hline $6 \mathbf{j}$ & 2,5-diclorofenila & $69,16 \pm 2,10$ & $90,31 \pm 13,28$ & $106,54 \pm 6,84$ \\
\hline $6 \mathbf{k}$ & 3,4-difluorofenila & $68,30 \pm 3,52$ & $76,56 \pm 7,11$ & $85,27 \pm 6,59$ \\
\hline 61 & 4-metilfenila & $75,44 \pm 0,93$ & $69,01 \pm 1,47$ & $71,34 \pm 3,63$ \\
\hline $6 \mathrm{~m}$ & 2-bromofenila & $85,08 \pm 6,67$ & $78,78 \pm 7,39$ & $77,13 \pm 4,65$ \\
\hline $6 n$ & 3-bromofenila & $82,38 \pm 0,52$ & $79,88 \pm 0,07$ & $18,93 \pm 5,30$ \\
\hline $7 a$ & Fenila & $79,44 \pm 9,18$ & $80,66 \pm 3,27$ & $20,85 \pm 7,92$ \\
\hline $7 b$ & 4-fluorofenila & $84,64 \pm 11,17$ & $81,15 \pm 7,69$ & $38,24 \pm 4,95$ \\
\hline $7 c$ & 4-clorofenila & $75,67 \pm 1,30$ & $76,62 \pm 2,09$ & $12,39 \pm 3,79$ \\
\hline 7d & 4-bromofenila & $79,77 \pm 21,94$ & $79,58 \pm 12,48$ & $82,13 \pm 8,98$ \\
\hline $7 e$ & 4-iodofenila & $88,20 \pm 16,48$ & $87,13 \pm 15,32$ & $13,04 \pm 3,80$ \\
\hline $7 f$ & 4-nitrofenila & $74,66 \pm 5,60$ & $81,99 \pm 3,95$ & $39,62 \pm 5,36$ \\
\hline $7 \mathrm{~g}$ & 4-metoxifenila & $87,93 \pm 19,11$ & $87,50 \pm 16,95$ & $89,80 \pm 12,08$ \\
\hline $7 \mathrm{~h}$ & 4-trifluorometoxifenila & $72,33 \pm 2,03$ & $75,77 \pm 6,90$ & $64,97 \pm 2,09$ \\
\hline $7 \mathbf{i}$ & 4-trifluorometila & $85,09 \pm 20,36$ & $79,39 \pm 9,67$ & $84,12 \pm 15,99$ \\
\hline $7 \mathbf{j}$ & 3,4-difluorofenila & $76,27 \pm 7,42$ & $70,58 \pm 5,84$ & $77,09 \pm 3,30$ \\
\hline $7 \mathbf{k}$ & 4-metilfenila & $80,39 \pm 10,62$ & $64,95 \pm 9,08$ & $27,88 \pm 6,49$ \\
\hline 71 & 2-bromofenila & $93,92 \pm 10,74$ & $90,74 \pm 5,61$ & $88,74 \pm 2,32$ \\
\hline $7 \mathrm{~m}$ & 3-bromofenila & $93,03 \pm 8,93$ & $88,54 \pm 8,41$ & $19,88 \pm 5,19$ \\
\hline
\end{tabular}

* Para os compostos 6a-6n, $\mathrm{n}=1$ e para os compostos $\mathbf{7 a - 7 m , ~}=3$; **Considerou-se $100 \%$ de viabilidade celular no tratamento controle com o veículo DMSO. 
Tabela 2. Valores de $\mathrm{IC}_{50}\left(\mu \mathrm{mol} \mathrm{L}{ }^{-1}\right)$ obtidos para as linhagens celulares HL60, Nalm6 e B16F10 tratadas com os compostos $\mathbf{6 n}, 7 \mathbf{7 a}, 7 \mathbf{c}, 7 \mathbf{7 e}, 7 \mathbf{m}$

\begin{tabular}{lccc}
\hline \multirow{2}{*}{ Composto } & \multicolumn{3}{c}{$\mathrm{IC}_{50}\left(\mu \mathrm{mol} \mathrm{L}{ }^{-1}\right)$} \\
\cline { 2 - 4 } 6n & $\mathrm{HL60}$ & $\mathrm{Nalm6}$ & $\mathrm{B} 16 \mathrm{~F} 10$ \\
$\mathbf{7 a}$ & $70,74 \pm 0,269$ & $66,30 \pm 3,451$ & $\mathrm{NA}$ \\
$\mathbf{7 c}$ & $75,83 \pm 4,752$ & $85,59 \pm 2,913$ & $179,7 \pm 3,090$ \\
$7 \mathbf{7}$ & $74,61 \pm 2,171$ & $\mathrm{NA}$ & $\mathrm{NA}$ \\
$\mathbf{7 m}$ & $74,54 \pm 6,554$ & $\mathrm{NA}$ & $\mathrm{NA}$ \\
\hline
\end{tabular}

$\mathrm{IC}_{50}$ :concentração dos compostos requerida para inibir $50 \%$ da viabilidade celular, determinada após 48 horas de tratamento. (NA) Compostos não ativos nas condições experimentais utilizadas.

7m) possuem como característica estrutural comum o tamanho da cadeia carbônica lateral do anel triazólico, que é comparativamente maior que a do composto $6 \mathbf{n}$.

Para a linhagem HL60, verifica-se que todos os compostos foram capazes de inviabilizar o crescimento celular, apresentando valores de $\mathrm{IC}_{50}$ abaixo de $100 \mu \mathrm{mol} \mathrm{L}{ }^{-1}$. No entanto, dentre os cinco compostos investigados, o composto $\mathbf{6 n}$ foi o de maior atividade, apresentando valor de $\mathrm{IC}_{50}$ igual a $70,74 \mu \mathrm{mol} \mathrm{L} \mathrm{L}^{-1}$. Para a linhagem Nalm6, observa-se que os compostos mais ativos, 6n e 7m, possuem como característica estrutural comum a presença do grupo 3-bromofenila. O composto $\mathbf{6 n}$, que possui menor comprimento da cadeia alifática conectando a porção eugenol à porção triazólica, apresentou maior efeito citotóxico contra esta linhagem celular $\left(\mathrm{IC}_{50}\right.$ igual a $\left.66,30 \mu \mathrm{mol} \mathrm{L}^{-1}\right)$. Por último, em relação à linhagem celular B16F10, a qual é refratária ao tratamento quimioterápico em geral, ${ }^{24}$ observa-se que apenas o composto 7a foi capaz de diminuir a viabilidade celular, caracterizando-se como derivado triazólico do eugenol que apresentou atividade citotóxica contra as três linhagens celulares investigadas.

\section{CONCLUSÃO}

A conversão do eugenol em derivados contendo porções 1,2,3-triazólicas e avaliação da citotoxicidade destes resultou na identificação de cinco derivados que significativamente reduziram a viabilidade da linhagem leucêmica mieloide HL60. A subsequente avaliação destes derivados mais ativos mostrou que três deles apresentaram atividade contra a linhagem leucêmica linfoide Nalm6 enquanto que apenas um derivado apresentou citotoxicidade contra a linhagem de melanoma metastático murino B16F10. Embora as atividades descritas sejam moderadas, acredita-se que derivados triazólicos do eugenol possam representar uma classe de compostos a serem explorados visando à obtenção de novos agentes quimioterápicos contra o câncer. Estudos neste sentido estão em andamento em nossos laboratórios.

\section{MATERIAL SUPLEMENTAR}

Espectros de RMN, no IV e de massas utilizados na caracterização dos compostos estão disponíveis em http://quimicanova.sbq.org.br, na forma de arquivo PDF, com acesso livre.

\section{AGRADECIMENTOS}

À Fundação de Amparo à Pesquisa do Estado de Minas Gerais (FAPEMIG) pelo apoio por intermédio do Programa de Apoio a Grupos Emergentes (PRONEM) e CEX-APQ-02957-17. À Coordenação de Aperfeiçoamento de Pessoal de Nível Superior (CAPES) pelo apoio por meio do Programa Nacional de Cooperação Acadêmica (PROCAD) e pela concessão de uma bolsa de estudos (PARG).

\section{REFERÊNCIAS}

1. Balanchandran, P.; Govindarajan, R.; Pharmacol. Res. 2005, 51, 19.

2. http://www.inca.gov.br/estimativa/2016/index.asp?ID=2, acessada em Fevereiro de 2018.

3. De Araujo, M. E. M. B.; Dissertação de Mestrado, Universidade de São Francisco, Brasil, 2012.

4. Costa-Lotufo, L. V.; Montenegro, R. C.; Alves, A. P. N. N.; Madeira, S. V. F.; Pessoa, C.; Moraes, M. E. A.; Moraes, M. O.; Rev. Virtual Quim. 2010, 2, 47.

5. Huang, Z.; Tong, Y.; Wang, J.; Hang, Y.; Cancer Cell Int. 2003, 3, 5.

6. Ghorab, M. M.; Al-Said, M.; Arch. Pharm. Res. 2012, 35, 987.

7. Harvey, A. L.; Drug Discov. Today 2008, 13, 894.

8. Newman, D. J.; Cragg, G. M.; J. Nat. Prod. 2016, 79, 629.

9. Dias, D. A.; Urban, S.; Roessner, U.; Metabolites 2012, 2, 303.

10. Cheuka, P. M.; Mayoka, G.; Mutai, P.; Chibale, K.; Molecules 2017, 22, 58.

11. Jaganathan, S. K.; Supriyanto, E.; Molecules 2012, 17, 6290.

12. Kamatou, G. P.; Vermaak, I.; Viljoen, A. M.; Molecules 2012, 17, 6953.

13. Singh, P.; Jayaramaiah, R. H.; Agawane, S. B.; Vannuruswamy, G.; Korwar, A. M.; Anand, A.; Dhaygude, V. S.; Shaikh, M. L.; Joshi, R. S.; Boppana, R.; Kulkarni,M. J.; Thulasiram, H. V.; Giri, A. P.; Sci. Reports 2016, 6, 18798.

14. Yoo, C. B.; Han, K. T.; Cho, K. S.; Ha, J.; Park, H. J.; Nam, J. H.; Kil, U. H.; Lee, K. T.; Cancer Lett. 2005, 225, 41.

15. Perrin, D. D.; Armarego, W. L.; Purification of Laboratory Chemicals, $3^{\text {th }}$ ed., Butterworth-Heinemann Ltd.: Londres, 1988, p. 340.

16. Tiew, K.-C.; Dou, D.; Teramoto, T.; Lai, H.; Alliston, K. R.; Lushington, G. H.; Padmanabhan, R.; Groutas, W. C.; Bioorg. Med. Chem. 2012, 20, 1213.

17. Borgati, T. F.; Alves, R. B.; Teixeira, R. R.; Freitas, R. P.; Perdigão, T. G.; Silva, S. F.; Santos, A. A.; Bastidas, A. J. O; J. Braz. Chem. Soc. 2013, 24, 953.

18. Meldal, M.; TornФe, C. W.; Chem. Rev. 2008, 108, 2952.

19. Hein, J. E; Fokin, V.V.; Chem. Soc. Rev. 2010, 39, 1302.

20. Moses, J. E.; Moorhouse, A. D.; Chem. Soc. Rev. 2007, 36, 1249.

21. Kolb, H. C.; Finn, M. G.; Sharpless, K. B.; Angew. Chem., Int. Ed. 2001, 40, 2004.

22. Worrell, B. T.; Malik, J. A.; Fokin, V. V.; Science 2013, 340, 457.

23. Wu, P.; Fokin, V. V.; Aldrichimica Acta 2007, 40, 7.

24. Tatman, D.; Mo, H.; Cancer Lett. 2002, 175, 129. 